\title{
Time resolved spectral behavior of bright BATSE precursors ${ }^{\star}$
}

\author{
D. Burlon ${ }^{1}$, G. Ghirlanda ${ }^{2}$, G. Ghisellini ${ }^{2}$, J. Greiner $^{1}$, and A. Celotti ${ }^{3}$ \\ 1 Max-Planck-Institut für Extraterrestrische Physik, Giessenbachstraße 1, 85740 Garching, Germany \\ e-mail: burlon@mpe.mpg.de \\ 2 Osservatorio Astronomico di Brera, via E. Bianchi 46, 23807 Merate, Italy \\ 3 SISSA, via Beirut 2-4, 34151 Trieste, Italy
}

Received 9 June 2009 / Accepted 24 July 2009

\begin{abstract}
Aims. Gamma ray bursts (GRBs) are sometimes preceded by dimmer emission episodes, called "precursors", whose nature is still a puzzle: they could either have the same origin as the main emission episode or they could be due to another mechanism. We investigate if precursors have some spectral distinctive feature with respect to the main GRB episodes.

Methods. To this aim we compare the spectral evolution of the precursor with that of the main GRB event. We also study if and how the spectral parameters, and in particular the peak of the $v F_{v}$ spectrum of time resolved spectra, correlates with the flux. This allows us to test if the spectra of the precursor and of the main event belong to the same correlation (if any). We searched GRBs with precursor activity in the complete sample of 2704 bursts detected by BATSE finding that $12 \%$ of GRBs have one or more precursors. Among these we considered the bursts with time resolved spectral analysis performed by Kaneko et al. (2006, ApJS, 166, 298), selecting those having at least two time resolved spectra for the precursor.

Results. We find that precursors and main events have very similar spectral properties. The spectral evolution within precursors has similar trends as the spectral evolution observed in the subsequent peaks. Also the typical spectral parameters of the precursors are similar to those of the main GRB events. Moreover, in several cases we find that within the precursors the peak energy of the spectrum is correlated with the flux similarly to what happens in the main GRB event. This strongly favors models in which the precursor is due to the same fireball physics of the main emission episodes.
\end{abstract}

Key words. gamma rays: bursts - radiation mechanisms: non-thermal - gamma rays: observations

\section{Introduction}

How does a gamma ray burst (GRB) behave before the onset of the main emission is a debated issue. The so-called "precursor" activity has been observationally addressed by e.g. Koshut et al. (1995) [hereafter K95], Lazzati (2005) [L05] and Burlon et al. (2008) [B08].

K95 searched in the Burst Alert and Transient Source Experiment (BATSE) sample for precursors defined as pulses with a peak intensity lower than that of the main GRB and separated from it by a quiescent phase at least as long as the duration of the main event. They found precursors in $\sim 3 \%$ out of a sample of GRBs detected by BATSE up to 1994 May. Their duration appeared weakly correlated with those of the main GRBs and on average shorter than that of the burst. The spectral properties of these precursors showed no systematic difference with respect to those of the main GRB event, being both softer and harder. However, the comparison of the spectral properties of the precursors and of the main event were based on the hardness ratio (HR) which is only a proxy of the real shape of burst spectra.

L05 searched for precursors as weak events preceding the BATSE trigger. He found, within a sample of 133 bright GRBs, that $\sim 20 \%$ showed precursor activity. These precursors were on average extremely dimmer than the main GRB event, and their durations are weakly correlated with that of the main event. In contrast with the results of K95, the precursors studied by L05 were softer than the main event. Also in this analysis, however,

$\star$ Figures 8 to 41 are only available in electronic form at http://www . aanda.org the spectral characterization of the precursors were based on the fluence HR. However, given the typically extreme low fluence of most of the precursors found by L05, a better spectral characterization (e.g. through model fits of a high resolution BATSE spectrum) was almost impossible. A difference is how the precursor-to-burst separation is measured: K95 consider the time difference between the peak of the precursor and that of the main event, while L05 measure the precursor-to-main event separation from the end of the precursor to the start of the GRB.

B08 searched for precursors in the sample of 105 Swift GRBs with measured redshifts. In $\sim 15 \%$ of the sample a precursor was found. The definition of precursors adopted in B08 is similar to that used by K95. The main difference, however, is that B08 did not require that the precursor precedes the main event by an amount of time comparable to the duration of the main event. The novelty of B08 was to search and study precursors found in a sample of bursts with known redshifts. This allowed, for the first time, to characterize the precursor energetics and to study how they compare with the main event energetics, also as a function of the rest-frame time separation between the precursors and the main events. The results of B08 suggest that precursors' spectra are consistent with those of the main event. Moreover, regardless of the rest-frame duration of the quiescence (i.e. the time interval separating the precursor and the burst), precursors carry a significant fraction of the total energy $(\approx 30 \%)$ of the main event (see Fig. 1 therein). The conclusions of B08 point to a common origin for both precursor and main event. Namely, they are nothing but two episodes of the same emission process. 
Theoretical models for precursors can be separated into three classes: the "fireball precursor" models (Mészáros \& Rees 2000; Ruffini et al. 2001; Daigne \& Mochkovitch 2002; Lyutikov \& Blandford 2003; Li 2007); the "progenitor precursor" models (Ramirez-Ruiz et al. 2002; Lazzati \& Begelman 2005) and the "two step engine" model (Wang \& Mészáros 2007 [W07]; Lipunova et al. 2009 [L09]). In the first class the precursor is associated to the initially trapped fireball radiation being released when transparency is reached. In the second class, based on the collapsar scenario, the precursor is identified with the interaction of a weakly relativistic jet with the stellar envelope. A strong terminal shock breaking out of the envelope is expected to produce transient emission. In both classes of models the precursors emission is predicted to be thermal, characterized by a blackbody spectrum. As for the third class in W07 the progenitor collapse leads to the formation of a neutron star whose emission would be responsible for the precursor, while the star shrinks; subsequent accretion onto the neutron star causes its collapse onto a black hole, originating the GRB prompt. Conversely, in L09 the precursor is produced when a collapsing "spinar" halts at the centrifugal barrier, whereas the main emission is due to a spin-down mechanism. Thus, in L09 accretion is not invoked in either steps.

One of the main limitations of K95 and L05 analyses is the poor spectral characterization of precursors. They used the HR, i.e. the ratio of the counts (or fluences reported in the BATSE catalog) measured over broad energy channels. However, it is well established that the broad band spectra of GRBs can be fitted by empirical models (e.g. Band et al. 1993) composed by low and high spectral power-laws with different slopes. The HR is only a proxy of the real spectral properties of GRB spectra (e.g. Ghirlanda et al. 2009), in particular for GRBs with vastly different $E_{\text {peak }}$. The other main limitation of these studies, based on the BATSE GRB catalog, is the lack of redshifts. Indeed, this motivated the study of B08 of Swift GRBs with precursors of known redshifts. Nonetheless, the spectral analysis of B08 of Swift-BAT spectra was limited by the narrow spectral range (15$150 \mathrm{keV})$ : most Swift spectra of precursors could be fitted by a single power-law (i.e. the peak energy of the $v F_{v}$ spectrum is unknown) and in all cases no time resolved spectral analysis of the precursor could be performed.

The latter point is particularly important: the information carried by the strong spectral evolution of GRB spectra (e.g. Ghirlanda et al. 2002; Ryde et al. 2005; Kaneko et al. 2006 [K06]) is completely averaged out when time integrated spectra are considered (integrated over the duration of the burst or over the duration of single emission episodes, like the precursor and the main event in B08). An interesting feature found by time resolved analysis of GRB spectra is that there could be a positive trend between the spectral peak energy $E_{\text {peak }}$ and the flux $P$ within single emission episodes of GRBs (Liang et al. 2004) [L04]. Interestingly, this trend appears similar (Firmani et al. 2009) [F09] to that found between the rest-frame GRB peak energies and their isotropic equivalent luminosities, when considering different GRBs with measured $z$ (i.e. so-called "Yonetoku" correlation; Yonetoku et al. 2004).

For these reasons we consider, in this paper, a still unanswered question: how does the spectrum of the precursor evolve and how does it compare with the evolution of the associated main event? In order to answer this question we compare the time evolution of the spectral parameters of precursors and main events. We also want to test if a possible correlation between the peak energy and the flux, i.e. $E_{\text {peak }}^{\text {obs }}-P$ within the precursors exists. If this correlation is due to the physics of the emission process or to that of the central engine is still to be understood, but if the precursors and the main event do follow a similar correlation, this would be another piece of the puzzle (in addition to the results of B08) suggesting that precursors are nothing else than the first emission episodes of the GRB. To this aims spectral data with high time and spectral resolution are necessary. BATSE provides the best data for this purpose.

The paper is organized as follows: in Sect. 2, we describe the sample selection and global properties; in Sect. 3, we present the spectral comparison between the precursor and the main event within single GRBs and we draw our conclusions in Sect. 4.

\section{The sample}

The Compton Gamma Ray Observatory satellite (CGRO) had on board the BATSE (Fishman et al. 1989), which provided the largest sample of GRBs, detected during the 9 yr lifetime. By applying different precursor definitions, K95 and L05 searched for BATSE bursts showing a precursor activity. A common feature of these studies is that a precursor is a peak separated (i.e. preceding) by a time interval and with a lower count rate with respect to the main GRB event.

The definition of a precursor is somewhat subjective and can easily bias the sample. L05, by excluding precursors that triggered BATSE selected the faintest precursors. K95 instead is missing precursors which can be closer than the duration of the rest of the burst. For these reasons, consistently with the definition adopted in B08, we adopted a definition of "precursor" as any peak with a peak flux smaller than the main prompt that follows it and that is separated from the main event by a quiescent period (namely, a time interval during which the background subtracted light curve is consistent with zero). We did not assume a priori that precursors can occur only in long GRBs (i.e. duration of the main emission episode be $>2 \mathrm{~s}$ in the observer's frame), albeit in B08 we found no short burst with a precursor. We adopted this "loose" definition in order to check, a posteriori, if distinguishing characteristics emerge in the analysis. This definition is subject to find more easily precursors events of the type of K95 in the BATSE sample. Since K95 limited the search to half of the BATSE sample (considering events between 910405 and 940529) and due to the slightly different precursor definition, we searched for precursors in the complete BATSE sample.

The final BATSE GRB sample ${ }^{1}$ contains 2704 GRBs. We found $2121 \mathrm{GRBs}$ out of 2704 total triggers for which there was a $64 \mathrm{~ms}$ binned light curve ${ }^{2}$ available. We inspected the background subtracted light curve of each GRB and found 264 GRBs $(12.5 \%)$ with a precursor. The majority (191) of GRBs showed one precursor, 48 showed double precursors, 19 showed three precursors, 5 showed four precursors and in only one case we found five precursors, according to our definition.

\subsection{Sample properties}

From the 64 ms BATSE light curves we calculated the duration of the precursor and main emission event for each of the 264 GRBs with precursors. The duration was defined as in the BATSE GRB catalog, i.e. T90. This corresponds to an integral measure, being the time interval containing the $90 \%$ (from $5 \%$ to

\footnotetext{
1 http://heasarc.gsfc.nasa.gov/docs/cgro/batse/BATSE_ Ctlg/basic.html

2 http://heasarc.gsfc.nasa.gov/docs/cgro/batse/ batseburst/sixtyfour_ms/index. pdfjasmihtml
} 


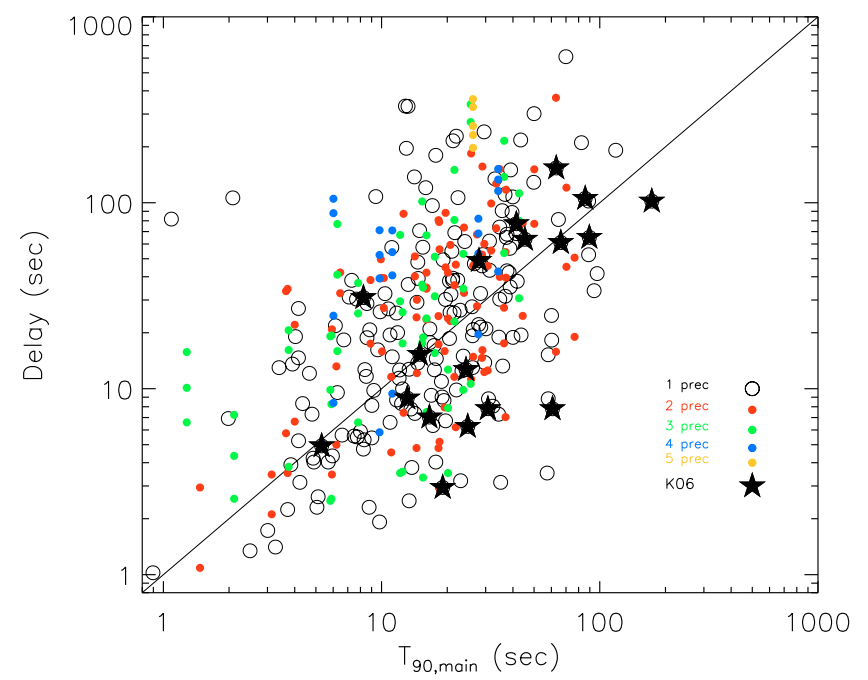

Fig. 1. Delay (precursors to main event) versus T90 of the main prompt emission for the 264 GRBs with precursors found in the BATSE sample. Black empty circles are GRBs with single precursors (191 cases), while filled dots show GRBs with multiple precursors (color code as in the legend). Black filled stars represent the 18 precursors with at least two spectra in K06. The solid line represents equality.

95\%) of the counts inside each peak considered, either precursor or main event.

We define the time delay between the precursor and the main event as the difference between the beginning of the main event and the end time of the precursor. The mean durations of precursors and main emission episodes are $\sim 15 \mathrm{~s}$ and $\sim 24 \mathrm{~s}$, respectively. The mean duration of the delays is $\sim 50 \mathrm{~s}$.

In Fig. 1, we show the delays of the precursors versus the duration T90 of the main GRB for the 264 GRB with precursors. The probability of a chance correlation among the duration of the GRBs with a single precursor (open circles and filled star symbols in Fig. 1) and the corresponding delay is $3.53 \times 10^{-14}$. An even lower chance probability is found including also GRBs with multiple precursors.

Since we do not know the redshift of these GRBs, we cannot exclude that the correlation is at least in part the result of the common redshift dependence of both the delay and the T90. Moreover, Fig. 1 shows no apparent difference between GRBs with single or multiple precursors. This result is somewhat different from that reported by Ramirez-Ruiz \& Merloni (2001). By investigating the temporal properties of multi-peaked GRBs (but note that they put no particular emphasis on precursors) they found a strong one-to-one correlation ( $4 \sigma$ consistency) between the duration of a peak and the duration of the quiescence time interval before it.

In Fig. 2, we show the ratio of the total counts (integrated over T90) of each precursor with respect to the counts in the corresponding main GRB plotted as a function of the delay time. In most cases the precursor total counts are a fraction (of the order $10-20 \%$ ) of the counts of the main GRB. Also in this case we do not find any difference between single precursors and multiple ones. Not surprisingly, a handful of GRBs show a precursor stronger than the main emission. In these cases, typically the precursor has a duration much larger than that of the main which over-compensates its lower peak flux, thus giving a higher integral count number for the precursor with respect to the main event.

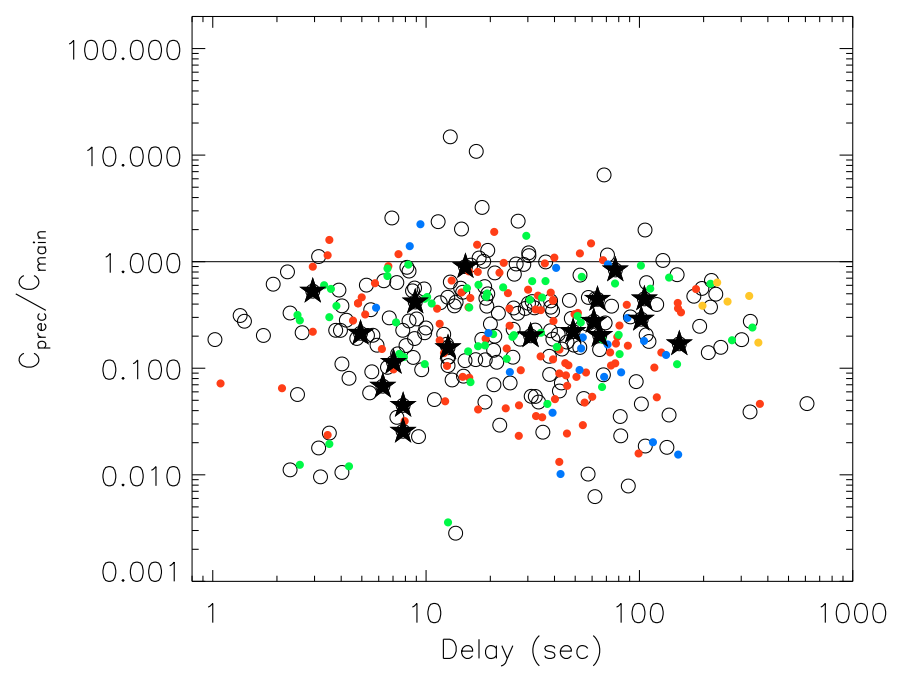

Fig. 2. Ratio of precursor to main event counts versus delay times. Symbols and color codes are as in Fig. 1.

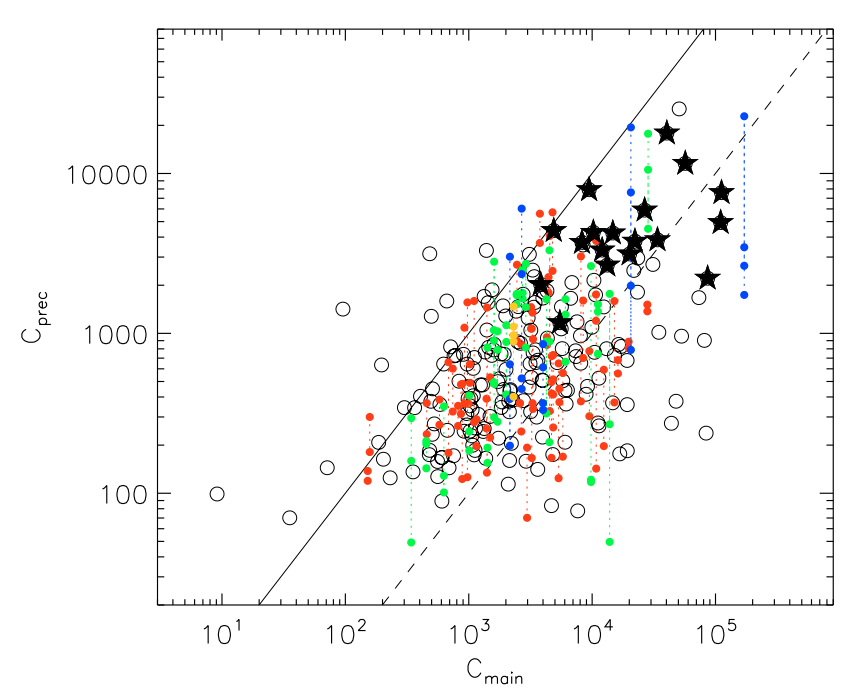

Fig. 3. Total counts of the precursor versus the total counts of main event. Symbols and color codes are as in Fig. 1. The solid line represents equality, while the dashed line corresponds to precursors dimmer than main events by a factor 10 .

Figure 3 shows the total counts of the precursors with respect to the total counts in the main GRBs. In this plane different selection cuts are evident. The selection criterion for defining precursors in this work is evident as the lack of precursors to the left of the equality line (solid).

It is apparent from Figs. 2 and 3 that neither the delay times of the precursors with respect to the onset of the main event, nor the integrated counts of the peaks seem to show a specific clustering. Therefore, we can rule out the existence of a subclass of "real" precursors among the complete sample, given the selection method.

\section{Spectral evolution}

In order to study the spectral evolution of the precursors and compare it with that of the main event, we rely on the time resolved 
spectral catalog of Kaneko et al. (2006). K06 analyzed the spectra of selected bright BATSE GRBs. These were selected to have a peak photon flux (on the $256 \mathrm{~ms}$ timescale and integrated in the $50-300 \mathrm{keV}$ ) greater than 10 photons $\mathrm{cm}^{-2} \mathrm{~s}^{-1}$ or a total energy fluence greater than $2.0 \times 10^{-5} \mathrm{erg} \mathrm{cm}^{-2}$ in the energy range $\sim 20-$ $2000 \mathrm{keV}$. This mixed criterion ensured K06 to have a minimum number of time resolved spectra distributed within the duration of each GRB so to study the features of its spectral evolution with sufficient details. This led to a sample of $350 \mathrm{GRB}$.

For most GRBs the high energy resolution data of the LAD detectors were analyzed. These data consists of $\sim 128$ energy channels distributed between $\sim 30 \mathrm{keV}$ and $2 \mathrm{MeV}$ accumulated during the burst with a minimum time resolution of $128 \mathrm{~ms}$. In some cases also lower energy resolution data (MER) were analyzed. K06 fitted both the time integrated spectra and the time resolved spectra with five different spectral models: a simple power-law (PWR), the Band model (Band et al. 1993) (BAND), a Band model with fixed high energy power-law component $\beta$ (BETA), a power-law with an exponential cutoff at high energies (COMP), or a smoothly broken power-law (SBPL). The spectra within a single GRB were accumulated in time according to a minimum $S / N$ ratio (required to be larger than 45 in each time resolved spectrum, integrated over the energy range 30 and $2000 \mathrm{keV}$ ). In the final catalog of K06 the best fit parameters for all the fitted models are given for all the time resolved spectra within a single burst. Through this large data set, it is possible to construct the time evolution of the spectral parameters of the bursts.

We cross-checked the sample of K06 with the 264 GRB with precursors that we have found in the BATSE catalog. We found 51 GRBs with precursors with time resolved analysis reported in the K06 sample. However, since our aim is to characterize how the spectrum of the precursor evolves in time, we restricted this sample to those GRBs with at least two time resolved spectra analyzed by K06 in the time interval of the precursor. This condition reduces the sample to 18 GRBs. All these have a single precursor in their light curve (except for trigger \#6472, that has two precursors). In Figs. 1-3, these 18 events are shown (star symbols): they correspond to the bright end of the distribution of count fluence of the precursors.

For these 18 GRBs with time resolved spectral analysis reported in K06 we show (panel (a) of Fig. 4, Fig. 8 and following even figures) the light curve in counts (and in physical units as obtained by the spectral analysis) and the time evolution of the best fit parameters. It has been shown that when analyzing time resolved BATSE spectra, especially for $S / N \lesssim 80$ (e.g. K06), the best fit model is often a cutoff power-law. This might be due to the difficulty of constraining the best fit parameter of the BAND model (i.e. the high energy spectral index of the powerlaw) when the fluence of the spectrum is low (as systematically expected in time resolved spectra with respect to time integrated ones). For this reason we decided to plot for all the 18 GRBs the spectral results given by K06 of the fit with the COMP model. In some cases this is not the best fit model of the time resolved spectra but for the aims of the present analysis, i.e. the relative comparison of the spectral evolution of precursors with respect to that of main bursts, any systematic effect due to the fit of the spectra with the COMP model is not affecting our conclusions. We show in Fig. 4 that both the photon spectral index and $E_{\text {peak }}^{\text {obs }}$ follow a strong soft-to-hard evolution in the rising part of the precursor, and vice versa in the descending part. In the main emission event both spectral parameters show a general hard-tosoft trend, but inside each peak they both follow the same trend

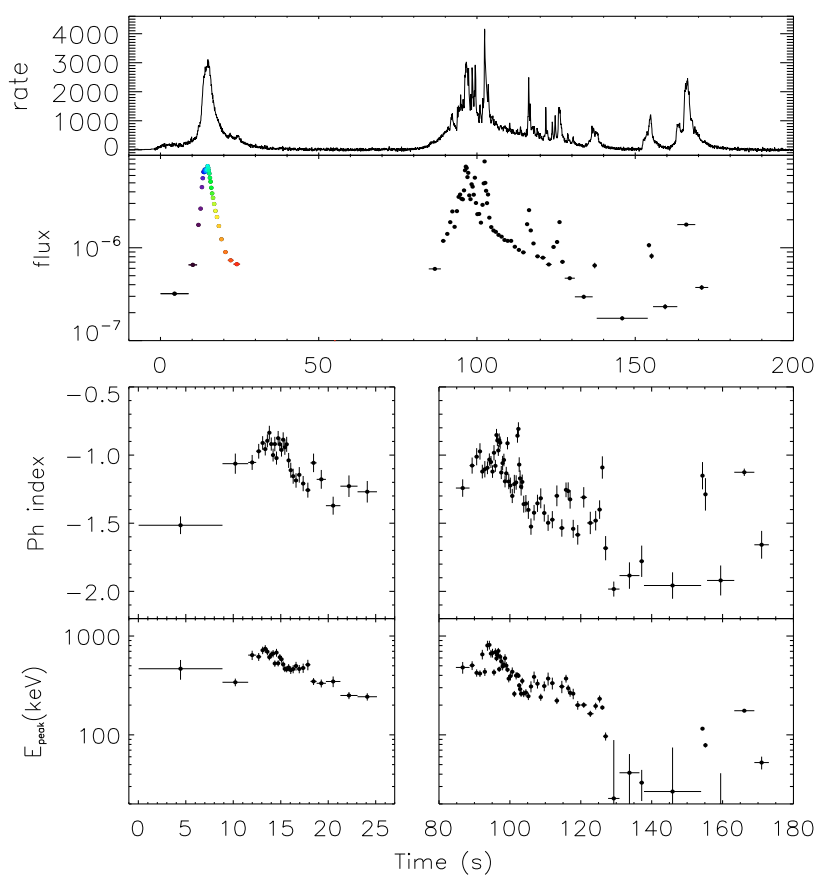

a) The top panel shows the complete light curve in units of count rate and just below is the same light curve in physical units $\left(\mathrm{erg} \mathrm{cm}^{-2} \mathrm{~s}^{-1}\right)$ binned into time intervals corresponding to the time resolved spectra extracted and analyzed by K06. The mid-panels show the evolution of the spectral parameters of the COMP model, i.e. the low energy photon spectral index $\alpha$ and the peak energy of the $v F_{v}$ spectrum $\left(E_{\text {peak }}\right)$. These correspond to the zoom in the time interval of the precursor and of the main event (color symbols corresponding to the precursor).

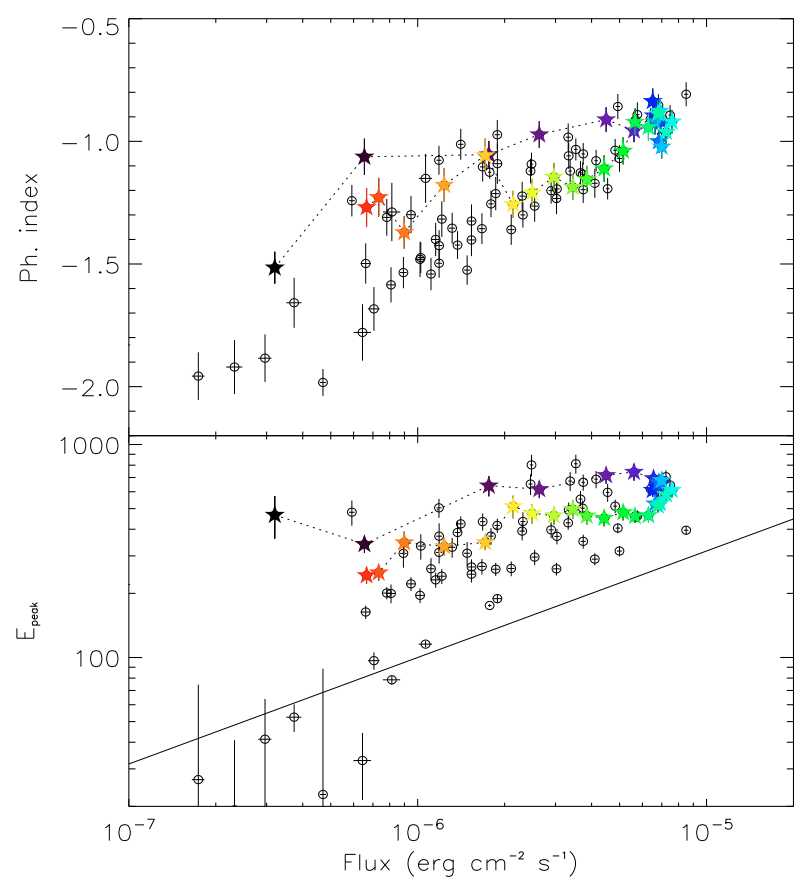

b) Panels show $\alpha$ (top) and $E_{\text {peak }}^{\text {obs }}$ (bottom) versus the flux. The spectral parameters of the precursor are shown with filled stars and joined by a dashed line. The first spectrum is the black one. The spectral parameters of the main emission episode are shown with empty circles.

Fig. 4. GRB 930201 (trigger \#2156). 
shown inside the single peak of the precursor and moreover they track the flux.

The latter consideration is shown in panel (b) of Fig. 4 (see for comparison the lower panel of Fig. 9 and following odd figures) where a correlation between the peak energy $E_{\text {peak }}^{\text {obs }}$ and the flux $P$ is apparent. Note however that GRB 930201 is the case with best statistics and hence does not necessarily stand for a general behavior. We connected (dashed line) the evolution of the spectral parameters only inside the precursor. The color code is as in panel (a): namely, the first (last) spectrum is the black (red) one. It has been recently pointed out (e.g. Borgonovo \& Ryde 2001; Liang et al. 2004, and more recently by Firmani et al. 2009 for Swift GRBs), that when considering the spectral evolution of long GRBs there is a trend between the evolution of the flux $P$ and the peak energy $E_{\text {peak }}^{\text {obs }}$ i.e. approximately $P \propto E_{\text {peak,obs }}^{\gamma}$. In particular Firmani et al. (2009) show that $84 \%$ of the K06 sample have $\gamma \sim 2$ at the $3 \sigma$ level. In addition, the correlation is not biased systematically by the value of $P$, though its uncertainty increases with decreasing flux. We can fiducially extrapolate this evidence to precursors, keeping open the question of identifying the hidden physical mechanism that determines the value of $\gamma$.

Intriguingly, this is similar to the correlation between the peak luminosity and the peak energy (time integrated over the duration of the burst) in GRBs with measured redshifts (socalled "Yonetoku" correlation). A similar result was reached by Liang et al. (2004) based on the spectral evolution of the brightest BATSE GRBs but for which no redshift was measured. Again, when studying the correlation between the luminosity and the peak energy within the few GRBs detected by BATSE and with known $z$, Firmani et al. (2009) finds that the correlation is present. The existence of a correlation within a single GRB similar to the Yonetoku correlation could be indicative of a physical origin for the quadratic link between the flux and the peak energy.

We can test if and how such a correlation holds in the GRBs with precursors that we have considered and/or if the $E_{\text {peak }}^{\text {obs }}$ and $P$ of the precursor are consistent with the correlation defined by the prompt.

If this correlation is due to the physics of the emission process or to that of the central engine is still to be understood, but if the precursors and the main event do follow a similar correlation, this would be another piece of the puzzle suggesting that precursors are nothing else than the first emission episodes of the GRB.

\section{Discussion}

Figure 5 shows the photon spectral indices $\alpha$ versus the peak energy $E_{\text {peak }}^{\text {obs }}$ for all 51 GRBs with precursor present in K06, while Fig. 6 shows for the same bursts how $\alpha$ and $E_{\text {peak }}^{\text {obs }}$ behave with the flux $P$. Different symbols (and colors, in the electronic edition) marks the precursor and the main event points. Filled symbols correspond to the $18 \mathrm{GRBs}$ with at least two spectra for the precursors. Red triangles mark the remaining precursors in K06 with just one spectrum. Empty black dots correspond to the spectral parameters of the main events.

Figure 5 shows that on average precursors and main GRB emission episodes span the same parameter space, while Fig. 6 shows that they follow similar correlations with the flux.

The distributions of the low energy photon indices $\alpha$ of the precursors and the main events are roughly consistent (the Kolmogorov-Smirnov KS null hypothesis probability is $\simeq 10^{-2}$ ).

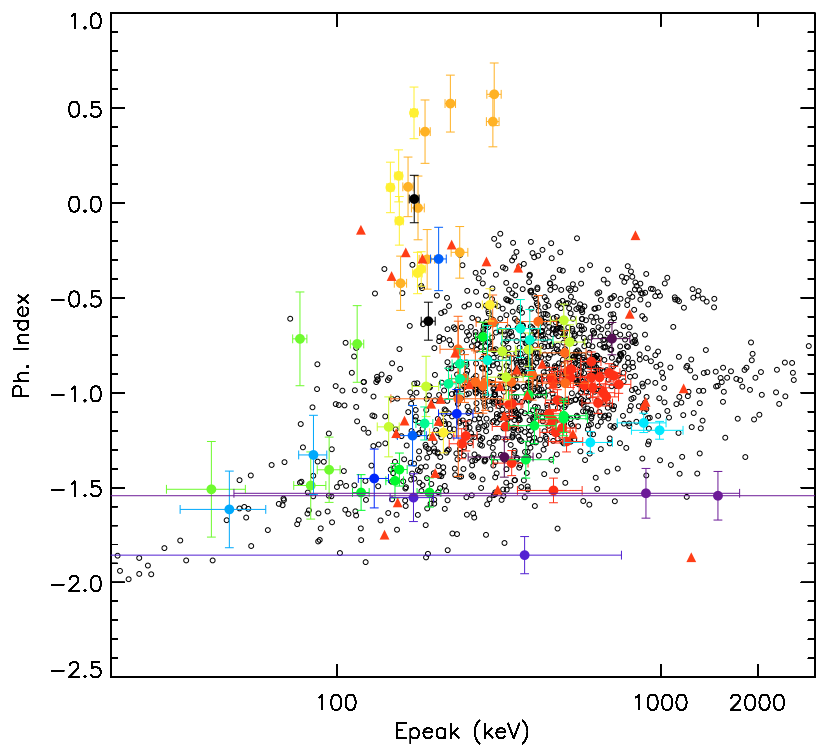

Fig. 5. Photon spectral indices $\alpha$ versus the peak energy $E_{\text {peak }}^{\text {obs }}$ for 51 GRBs with precursor. Spectral parameters correspond to the time bins of the time resolved spectra extracted and analyzed by K06. The 18 bursts belonging to our sample are shown with filled dots (different colors represent different GRBs). We added also 33 precursors with a single spectrum data point (red triangles). Spectral parameters of the main emission episode are shown in black empty circles.

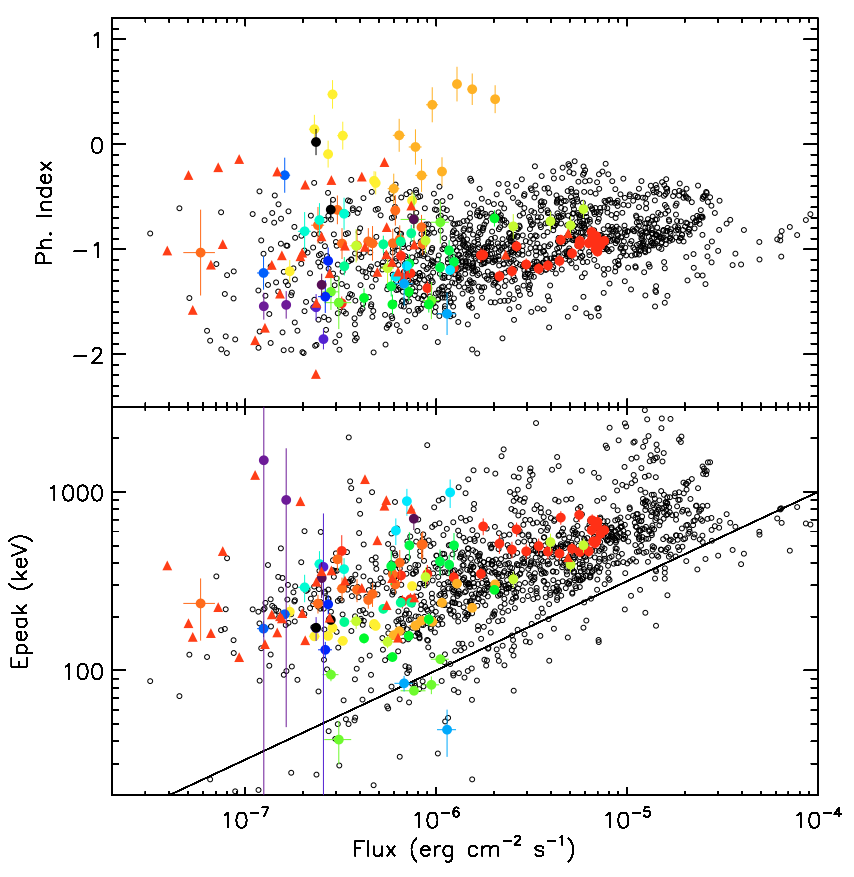

Fig. 6. The photon spectral index $\alpha$ (top) and $E_{\text {peak }}^{\text {obs }}$ (bottom) versus the flux $P$ in erg $\mathrm{cm}^{-2} \mathrm{~s}^{-1}$. The solid line in the bottom panel corresponds to $P \propto E_{\text {peak,obs. }}^{2}$. Color code and symbols as in Fig. 5.

Fitting the two distributions (see Fig. 7, upper panel) with Gaussian profiles we find $\left\langle\alpha_{\text {prec }}\right\rangle=-1.03 \pm 0.27$ and $\left\langle\alpha_{\text {main }}\right\rangle=$ $-0.94 \pm 0.34$.

Three (\#5486, \#6472, \#7343) of the 18 GRBs studied here present extremely hard spectra. One of them, i.e. GRB 960605 (\#5486, see Fig. 11), could even be consistent with a black-body 

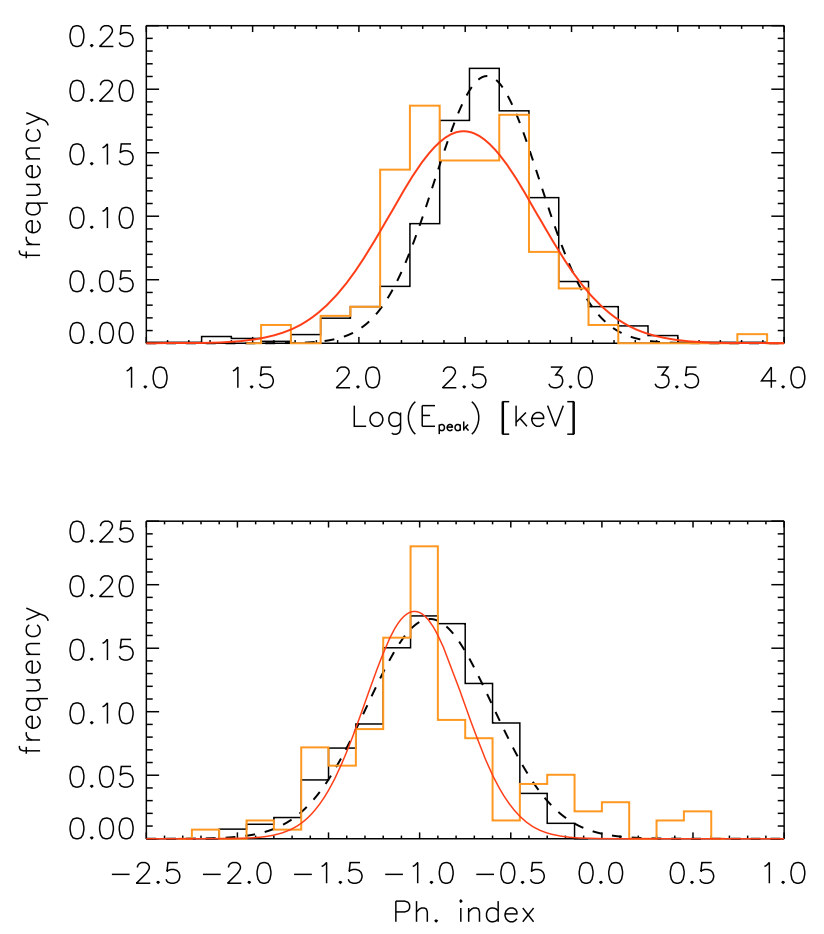

Fig. 7. Normalized distribution of $E_{\text {peak }}^{\text {obs }}(t o p)$ and of the spectral photon indices $\alpha$ (bottom) of precursors and main emission episodes. Precursor distribution and Gaussian fit are shown in colored filled line, main emission episode in black dashed line.

spectrum at the very beginning of the precursor. These few cases populate the upper part of Figs. 5 and 6 (upper panel). We have re-extracted the LAD data for this burst and reanalyzed them. We confirm the findings of K06. The finding of a precursor with a spectrum consistent with a black-body should not be taken as a proof of a radical difference with the main event, since it has been already pointed out (e.g. Ghirlanda et al. 2003) that a non-negligible fraction of GRB $(\sim 5 \%)$ start their emission with a black-body spectrum.

Comparing the distributions of $\log \left(E_{\text {peak }}^{\text {obs }}\right)$ we find that they are somewhat different (K-S null hypothesis probability $\sim 10^{-4}$ ). Fitting again with Gaussian profiles the two distributions in Fig. 7 (lower panel) we find the mean value and $1 \sigma$ scatter for precursors: $\log \left(E_{\text {peak }}^{\text {obs }}\right)=2.49 \pm 0.35$ to be compared to $\log \left(E_{\text {peak }}^{\text {obs }}\right)=2.60 \pm 0.24$ for the main emission events. The distribution of $E_{\text {peak }}^{\mathrm{obs}}$ for the precursors is slightly softer than the one of the main prompt emission. This result is not surprising when looking at the bottom panel of Fig. 6: the peak energy of precursors seem to follow the trend (when $E_{\text {peak }}^{\text {obs }}$ is plotted with respect to flux) drawn by the GRB main emission, but at the lower left end of the track.

In the 7 precursors with more time resolved spectra (\#2156, \#7688, \#5486, \#6472, \#3481, \#3241, \#1676), $E_{\text {peak }}^{\text {obs }}$ shows a strong evolution but nonetheless is always consistent with the correlation drawn by the main event (as shown in Fig. 4b and lower panels in Figs. 9, 11, 13, 15, 17, 19). Note that these similar trends in the evolution of $E_{\text {peak }}^{\text {obs }}$ do not depend upon the delay, as these vary among $\sim 9 \mathrm{~s}$ (for \#1676) and $\sim 75 \mathrm{~s}$ (for \#7688). Note that at odds with B08, this consideration is based only on observed time intervals, because the redshift $z$ is unknown for all GRBs in this work. Two of them, namely \#2156 and \#1676, also show consistent evolution in $\alpha$ between the precursor and the main event (see upper panels of Figs. 4b and 19). The other 5 GRBs (of this group of 7) show an evolution in $\alpha$ which is different in the precursor and in the main event: in two cases (\#5486 and \#6472) $\alpha$ starts extremely hard and evolves to softer values (see upper panel of Figs. 11 and 13). In the last three cases (\#3481, \#3241 and \#7688) either the photon spectral index evolves in a different way with respect to the one of the main emission episode (as in Figs. 17 and 9, upper panels), or it lies in a different region of the parameter space (see upper panel of Fig. 15).

The remaining 11 GRBs of our sample have more coarsely sampled precursor spectra. The trend of $E_{\text {peak }}^{\text {obs }}$ of the precursor is consistent with that of the main event in eight cases. In \#3253, \#6454, \#3057, \#4368, \#1157, \#6629, \#3301, \#7343 (see upper panels of Figs. 23, 25, 27, 29, 33, 35, 39 and 41) the peak energy in the spectra of precursors follow the same correlation with the flux drawn by the main emission. Notwithstanding, the number of spectra extracted by K06 in the precursor varies between five and two, thus preventing any more confident claim. Among these 8 GRBs, in the latter 3 the photon spectral indices $\alpha$ of the precursors do not track the trend drawn by the main emission event (see upper panels of Figs. 35, 39, and 41), being always softer (with the exception of the onset of the precursor in \#7343, which has $\alpha \simeq 0$ ). In the former badly sampled 5 GRBs, also $\alpha$ is consistent with the trend described by the spectra of the main impulse. Note that also in these eight cases the delay does not represent a distinguishing feature, as it can vary from $7 \mathrm{~s}$ (e.g. \#3253) up to $>100 \mathrm{~s}$ (\#3663).

The last three GRBs, namely \#3663, \#2700, and \#3448 present hardly distinguishable spectral characteristics (i.e. both $\alpha$ and $\left.E_{\text {peak }}^{\text {obs }}\right)$. This is due either to the extremely low number of spectra extracted in the precursor, or in the main impulse, or both at the same time (see Figs. 21, 31, and 37). In our opinion this prevents any further claim.

\section{Conclusion}

In this work we presented, for the first time, a time resolved spectral analysis of bright precursors based on spectral parameters, namely the photon spectral indices $\alpha$ and the observed peak energy $E_{\text {peak }}^{\text {obs }}$. This was done by using high energy resolution spectra extracted by K06 in a sample of 350 bright GRBs out of the complete sample of 2704 confirmed GRBs observed by the BATSE instrument. Of the 51 bursts with precursor present in K06, we selected a sample of 18 GRBs having at least two time resolved spectra of the precursor.

The comparison with the main emission episode has three outcomes. The first is that the photon spectral indices of precursors and main events are consistent, while the peak energies of the precursors are mildly softer (see Fig. 7). Secondly, both $\alpha$ and $E_{\text {peak }}^{\mathrm{obs}}$ do show an evolution (extreme in a handful of cases) that defines a relation between the flux $P$ and the spectral parameters (note that the $P-E_{\text {peak,obs }}^{\gamma}$ correlation was recently reported (e.g. F09) regardless the presence of precursors). Finally, we showed that delays do not represent a distinguishing feature in the trend of $\alpha$ or $E_{\text {peak }}^{\text {obs }}$.

We found one GRB (out of 18) in which the onset of the emission of the precursor is consistent with black-body emission (i.e. \#5486 - see Fig. 11). This was expected, since Ghirlanda et al. (2003) showed that $5 \%$ of BATSE GRBs 
show extremely hard emission at the onset of the first impulse.

Moreover, comparing the integrated counts in the peaks of precursors with respect to the ones of the main impulses, we confirmed the results of B08 (see Fig. 3). Indeed precursors carry a significant fraction of the energy of the main emission episode, regardless the duration of the time interval of quiescence.

These results, in addition to B08, point strongly to the conclusion that the onset of emission of GRBs (called precursor), even if separated from the main emission episode by hundreds of seconds (in the observers frame), is indistinguishable from that of the main event. Moreover, the delay remains a puzzling issue. This suggests that we should reconsider the idea of what a precursor is. Since our result is partially in contrast with L05 we cannot rule out the possibility that "real precursors" belong to another class of very dim pulses of different origin. Nonetheless, both kind of precursors can show very long delays, thus tackling any theoretical model for GRB prompt emission.

Acknowledgements. We acknowledge Marco Nardini and Lara Nava for stimulating discussion. D. B. is supported through DLR 50 OR 0405. This research was partially supported by ASI-INAF I/088/06/0 and MIUR. We acknowledge the use of public data from the BATSE data archive. D. B. thanks the OAB for the kind hospitality during the completion of this work.

\section{References}

Band, D., Matteson, J., Ford, L., et al. 1993, ApJ, 413, 281

Borgonovo, L., \& Ryde, F. 2001, ApJ, 548, 770

Burlon, D., Ghirlanda, G., Ghisellini, G., et al. 2008, ApJ, 685, L19

Daigne, F., \& Mochkovitch, R. 2002, MNRAS, 336, 1271

Firmani, C., Cabrera, J. I., Avila-Reese, V., et al. 2009, MNRAS, 393, 1209

Fishman, G. J., Meegan, C. A., Wilson, R. B., et al. 1989, in Developments in Observations and Theory for Solar Cycle, Vol. 22, ed. R. M. Winglee, \& B. R. Dennis, 96

Ghirlanda, G., Celotti, A., \& Ghisellini, G. 2002, A\&A, 393, 409

Ghirlanda, G., Celotti, A., \& Ghisellini, G. 2003, A\&A, 406, 879

Ghirlanda, G., Nava, L., Ghisellini, G., Celotti, A., \& Firmani, C. 2009, A\&A, 496, 585

Kaneko, Y., Preece, R. D., Briggs, M. S., et al. 2006, ApJS, 166, 298

Koshut, T. M., Kouveliotou, C., Paciesas, W. S., et al. 1995, ApJ, 452, 145

Lazzati, D. 2005, MNRAS, 357, 722

Lazzati, D., \& Begelman, M. C. 2005, ApJ, 629, 903

Li, L.-X. 2007, MNRAS, 380, 621

Liang, E. W., Dai, Z. G., \& Wu, X. F. 2004, ApJ, 606, L29

Lipunova, G. V., Gorbovskoy, E. S., Bogomazov, A. I., \& Lipunov, V. M. 2009 [arXiv: 0903 . 3169]

Lyutikov, M., \& Blandford, R. 2003 [arXiv: astro-ph/0312347]

Mészáros, P., \& Rees, M. J. 2000, ApJ, 530, 292

Ramirez-Ruiz, E., \& Merloni, A. 2001, MNRAS, 320, L25

Ramirez-Ruiz, E., MacFadyen, A. I., \& Lazzati, D. 2002, MNRAS, 331, 197

Ruffini, R., Bianco, C. L., Fraschetti, F., Xue, S.-S., \& Chardonnet, P. 2001, ApJ, $555, \mathrm{~L} 113$

Ryde, F., Kocevski, D., Bagoly, Z., Ryde, N., \& Mészáros, A. 2005, A\&A, 432, 105

Wang, X.-Y., \& Mészáros, P. 2007, ApJ, 670, 1247

Yonetoku, D., Murakami, T., Nakamura, T., et al. 2004, ApJ, 609, 935 
D. Burlon et al.: Time resolved spectral behavior of bright BATSE precursors, Online Material $p 1$

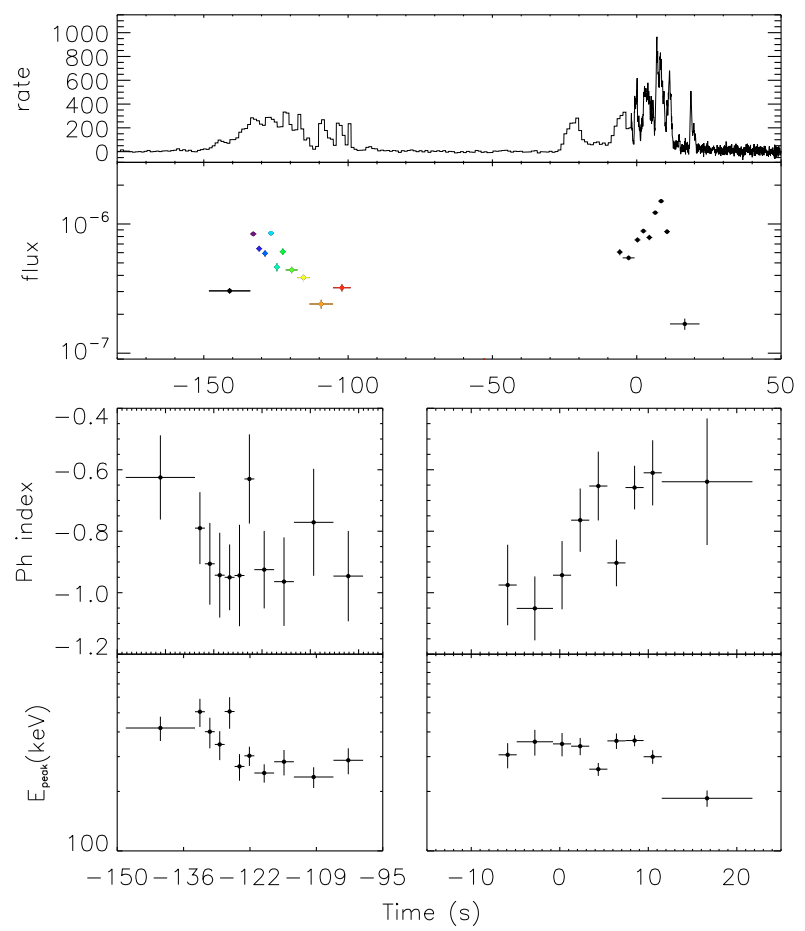

Fig. 8. Trigger \#7688. Color code and description as in Fig. 4a.

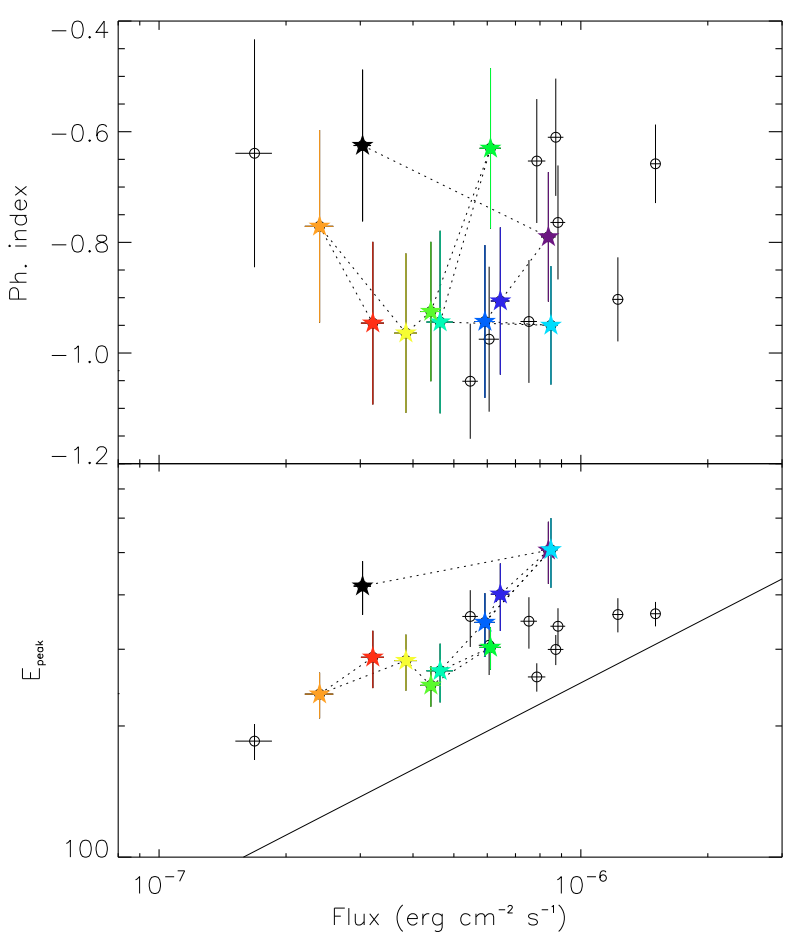

Fig. 9. Trigger \#7688. Color code and description as in Fig. 4b.
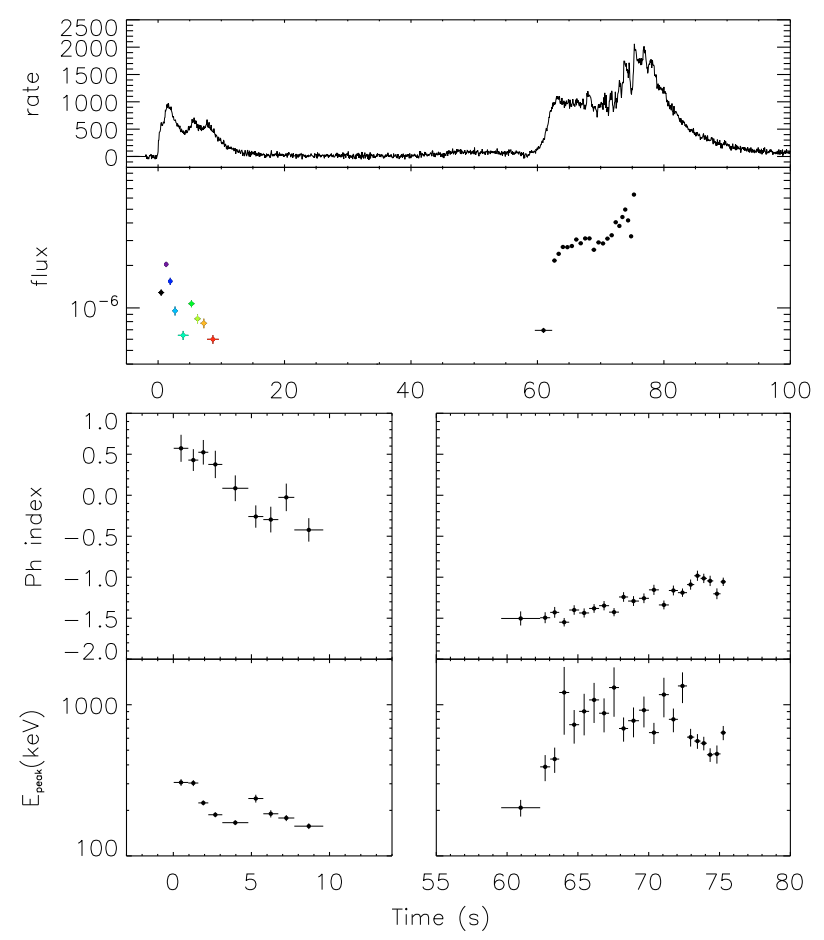

Fig. 10. Trigger \#5486. Color code and description as in Fig. 4a.

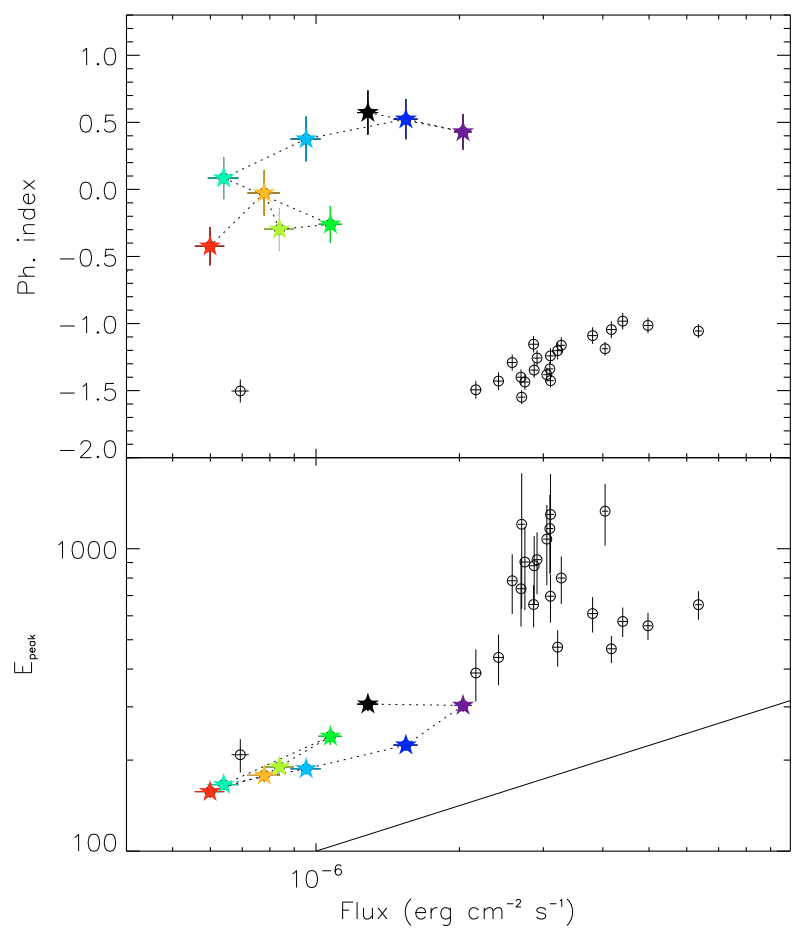

Fig. 11. Trigger \#5486. Color code and description as in Fig. 4b. 
D. Burlon et al.: Time resolved spectral behavior of bright BATSE precursors, Online Material p 2
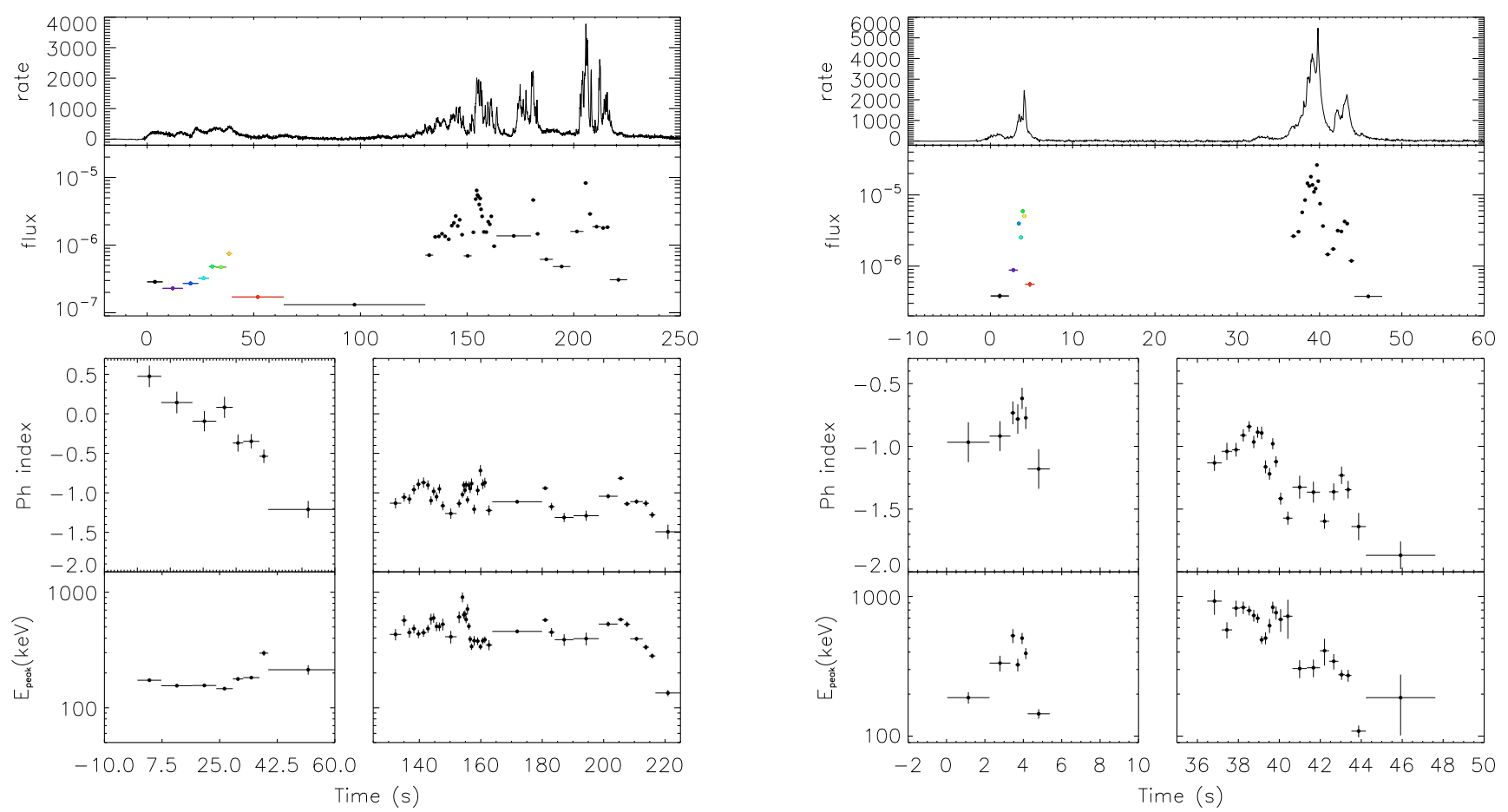

Fig. 12. Trigger \#6472. Color code and description as in Fig. 4a.

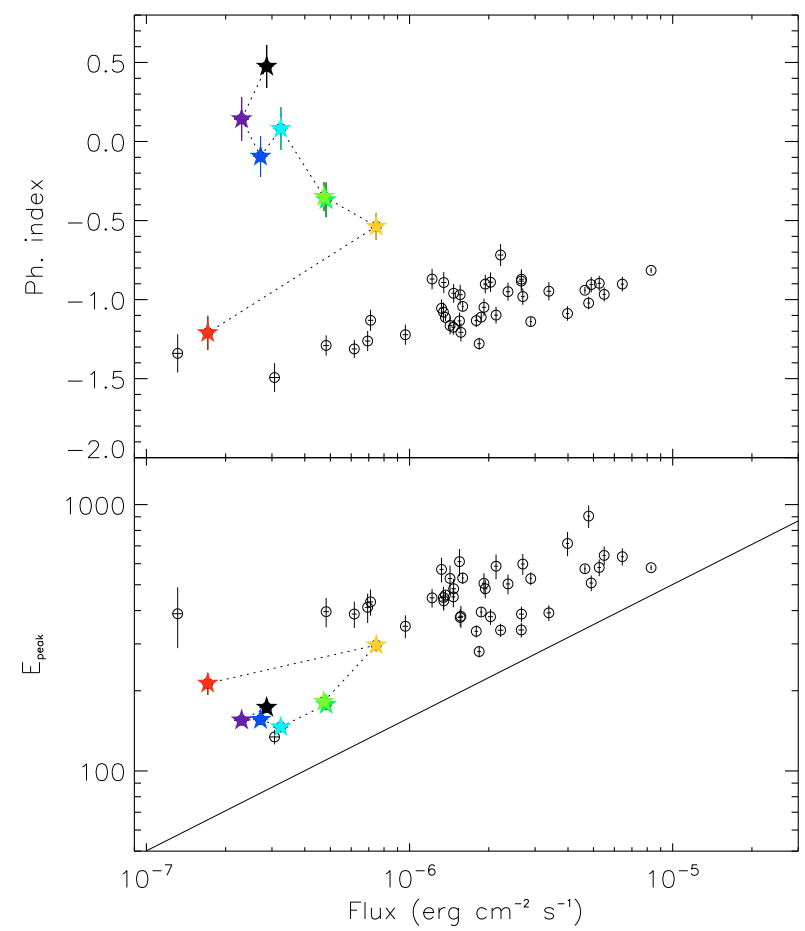

Fig. 13. Trigger \#6472. Color code and description as in Fig. 4b.

Fig. 14. Trigger \#3481. Color code and description as in Fig. 4a.

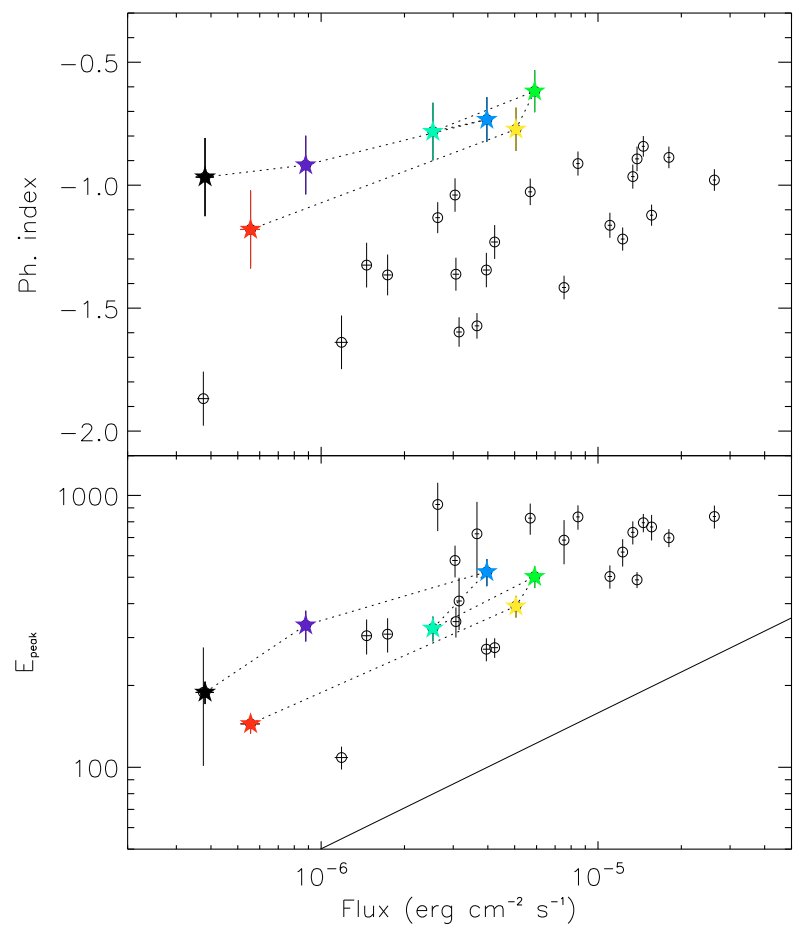

Fig. 15. Trigger \#3481. Color code and description as in Fig. 4b. 
D. Burlon et al.: Time resolved spectral behavior of bright BATSE precursors, Online Material p 3

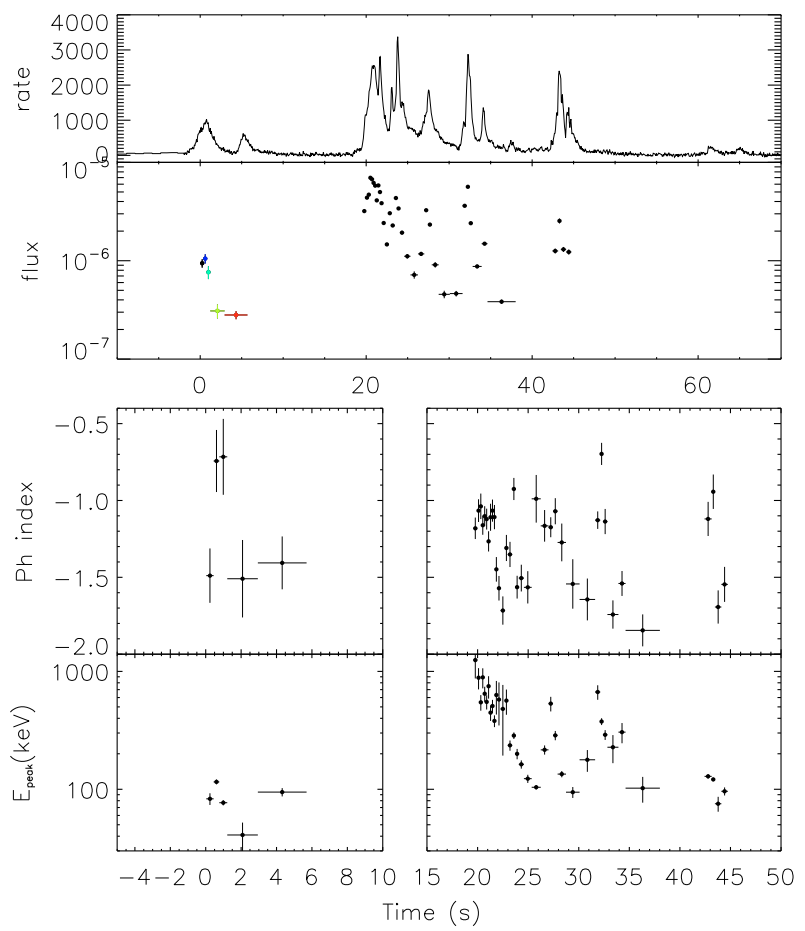

Fig. 16. Trigger \#3241. Color code and description as in Fig. 4a.

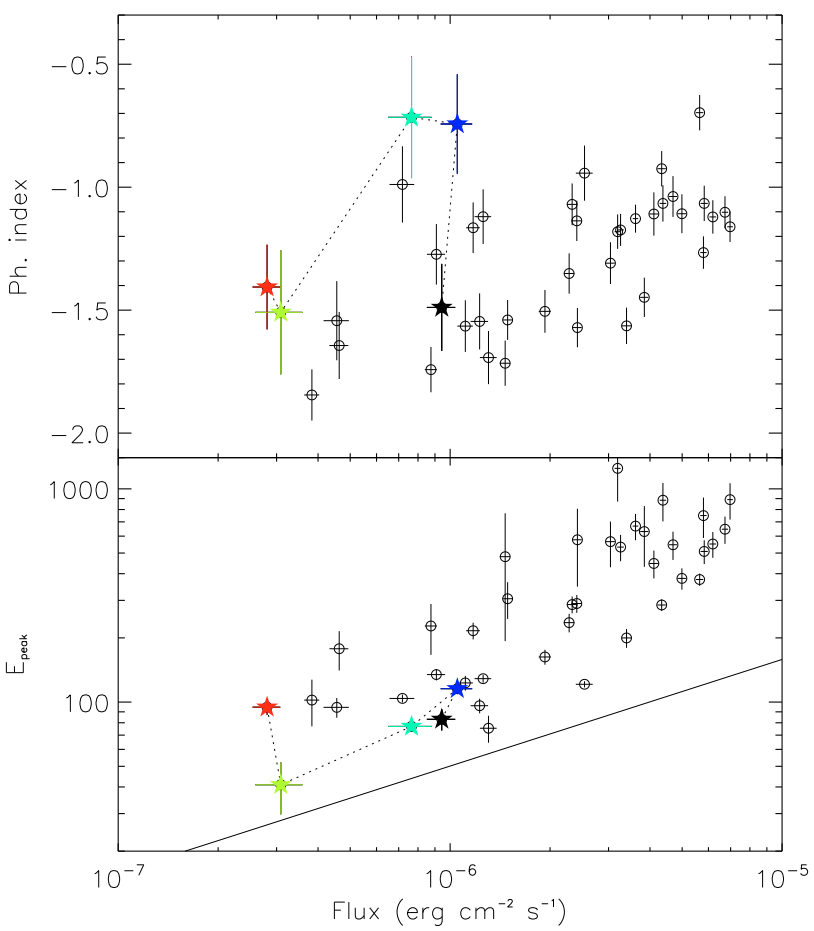

Fig. 17. Trigger \#3241. Color code and description as in Fig. 4b.

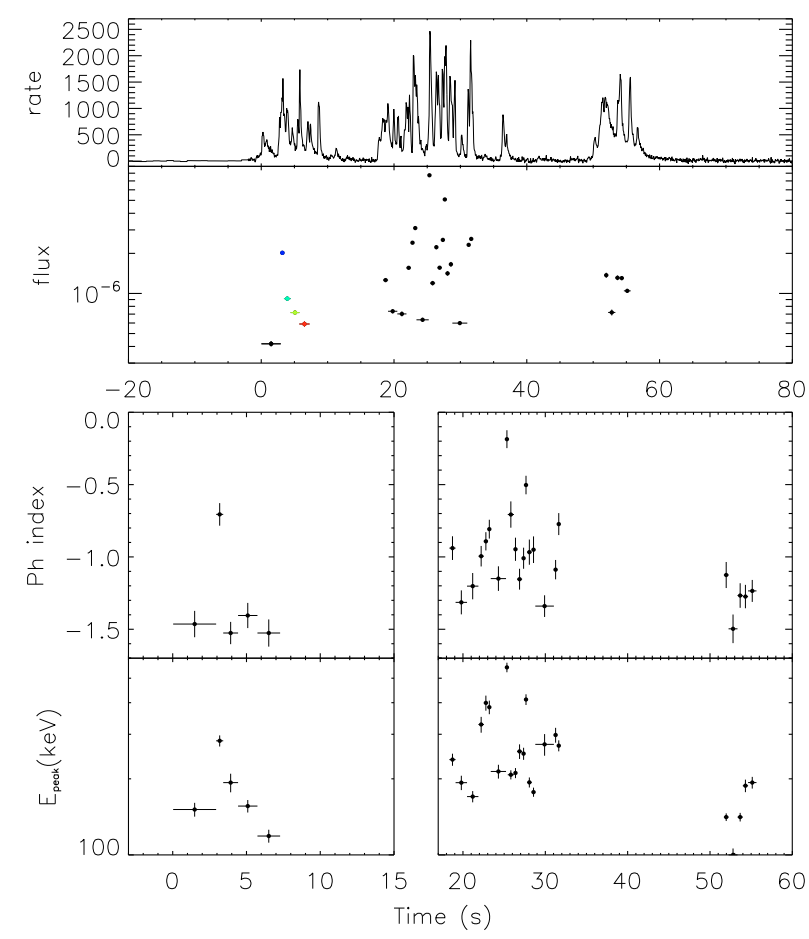

Fig. 18. Trigger \#1676. Color code and description as in Fig. 4a.

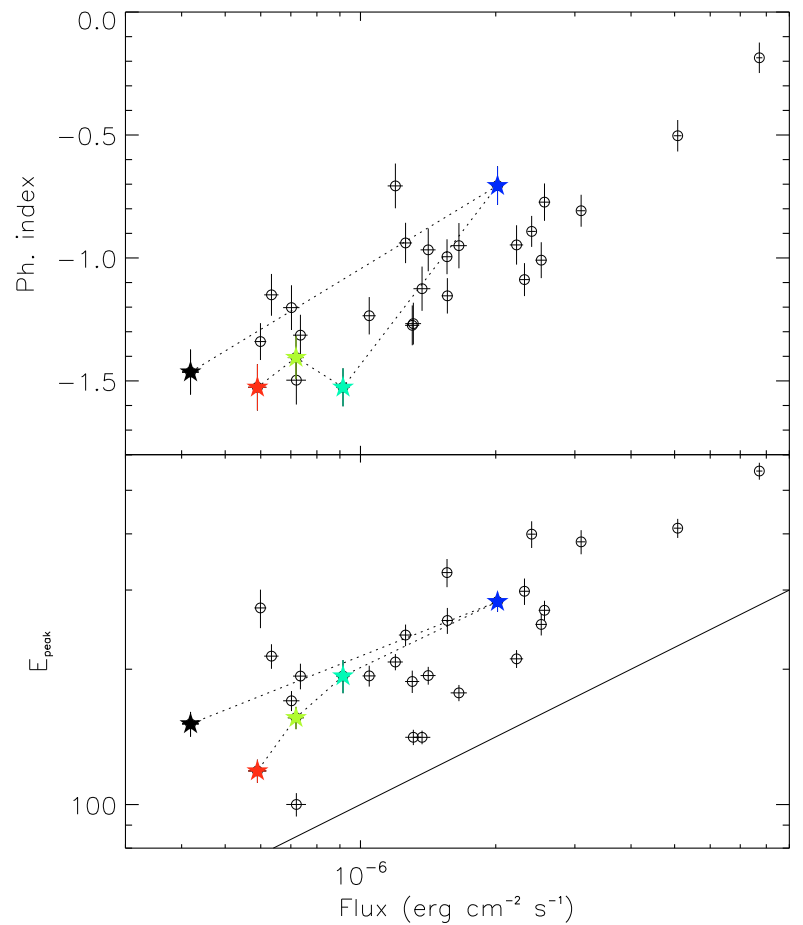

Fig. 19. Trigger \#1676. Color code and description as in Fig. 4b. 
D. Burlon et al.: Time resolved spectral behavior of bright BATSE precursors, Online Material $p 4$
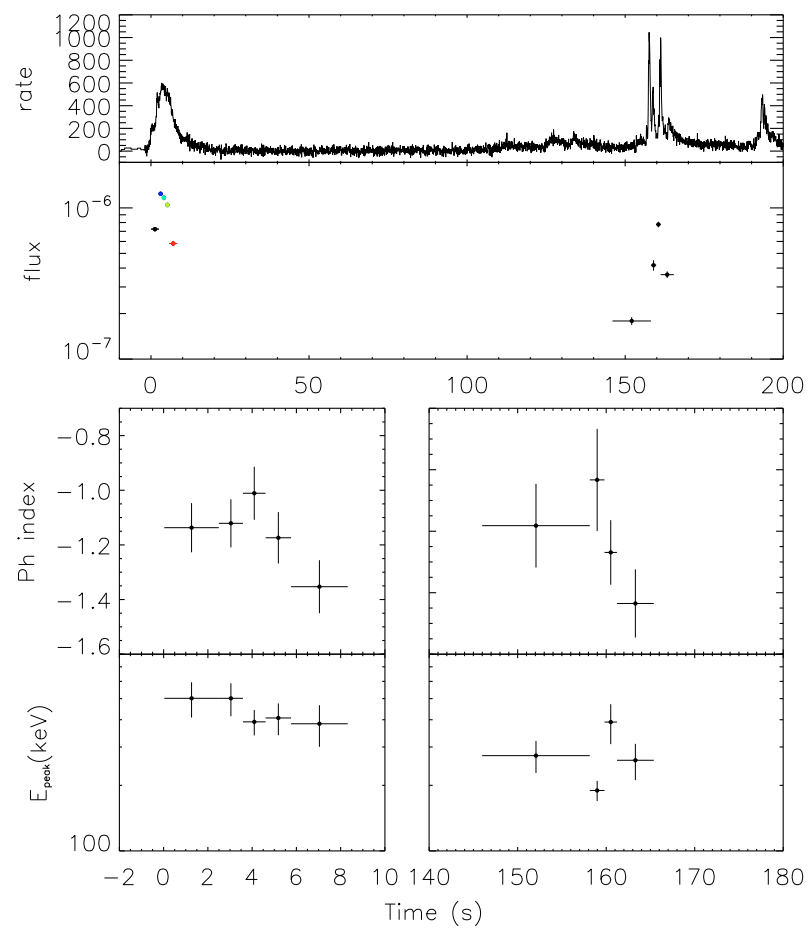

Fig. 20. Trigger \#3663. Color code and description as in Fig. 4a.

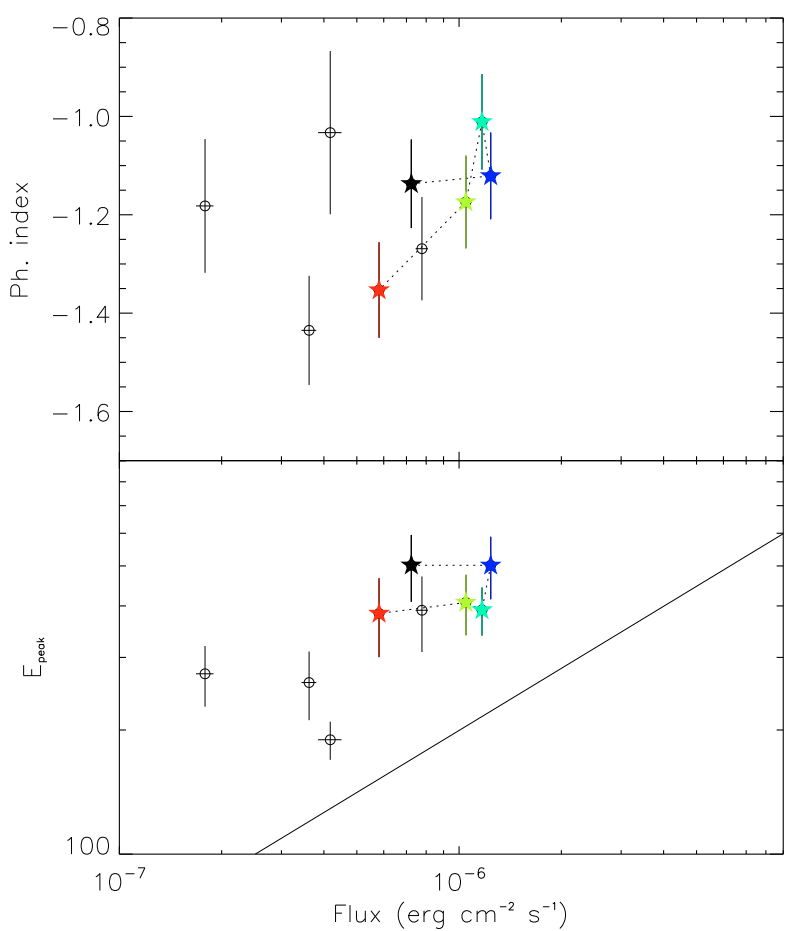

Fig. 21. Trigger \#3663. Color code and description as in Fig. 4b.
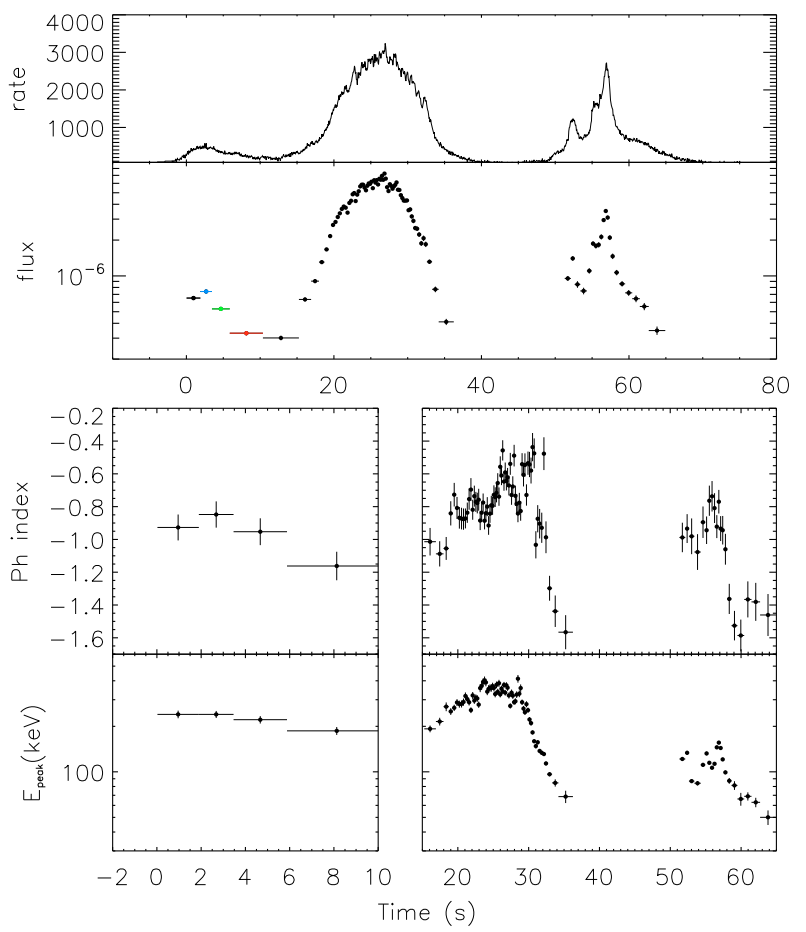

Fig. 22. Trigger \#3253. Color code and description as in Fig. 4a.

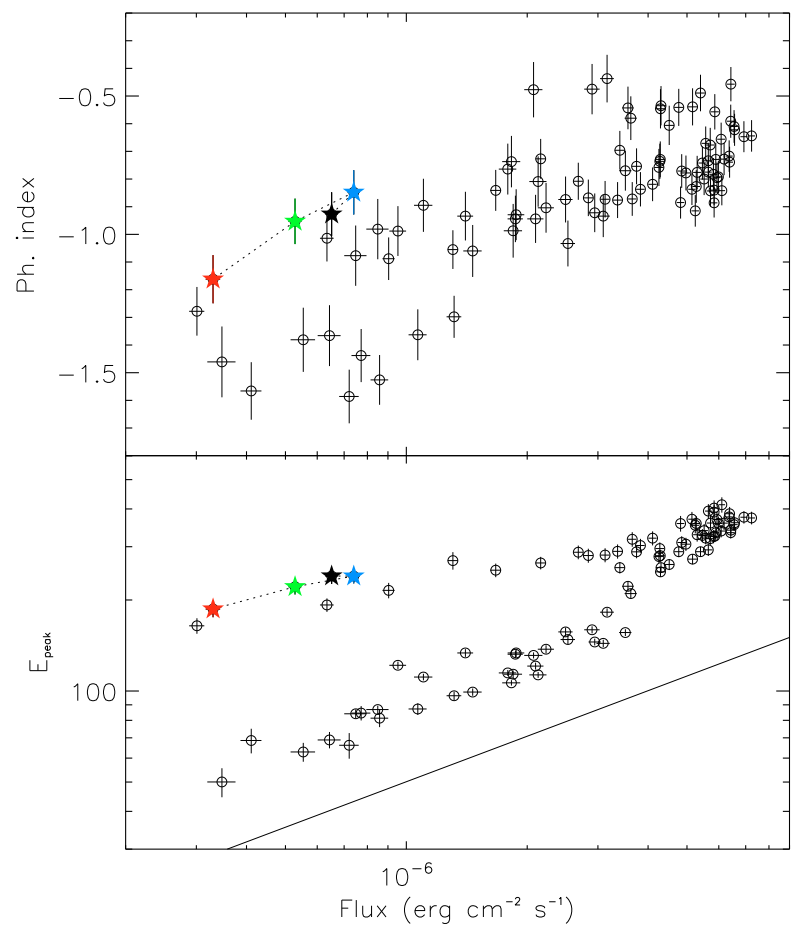

Fig. 23. Trigger \#3253. Color code and description as in Fig. 4b. 
D. Burlon et al.: Time resolved spectral behavior of bright BATSE precursors, Online Material p 5

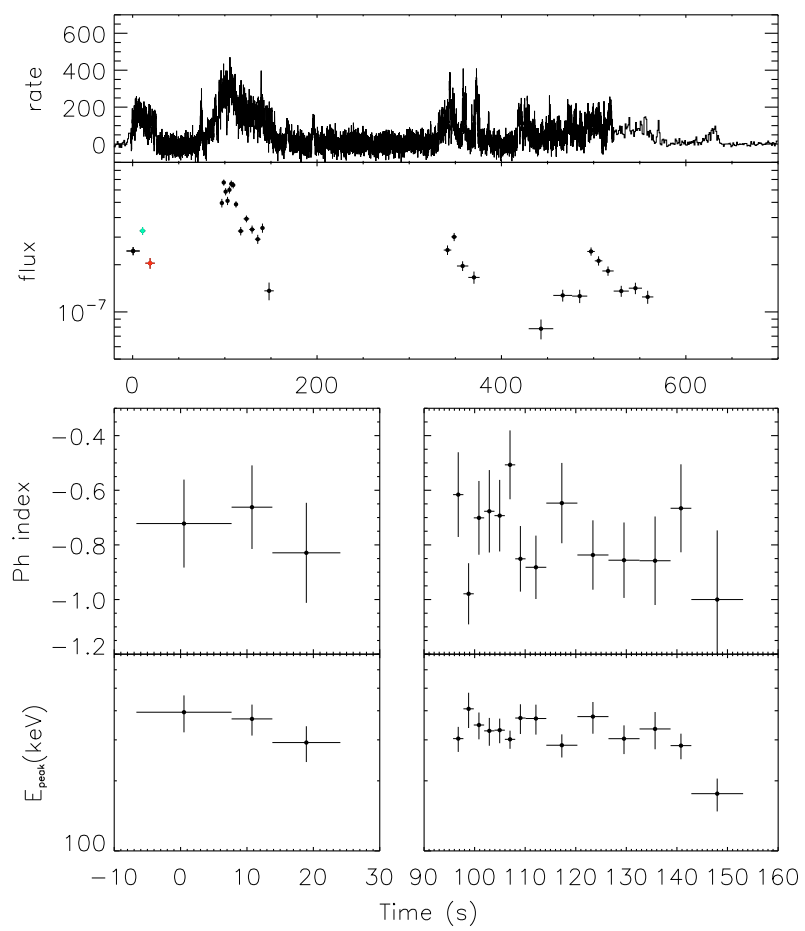

Fig. 24. Trigger \#6454. Color code and description as in Fig. 4a.

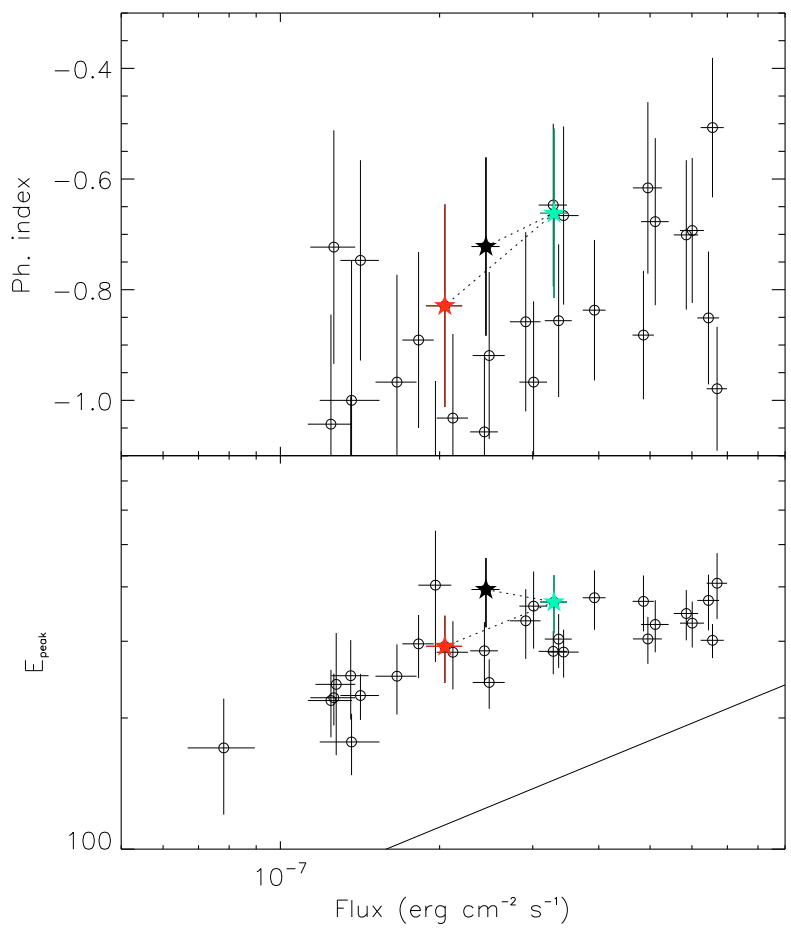

Fig. 25. Trigger \#6454. Color code and description as in Fig. 4b.

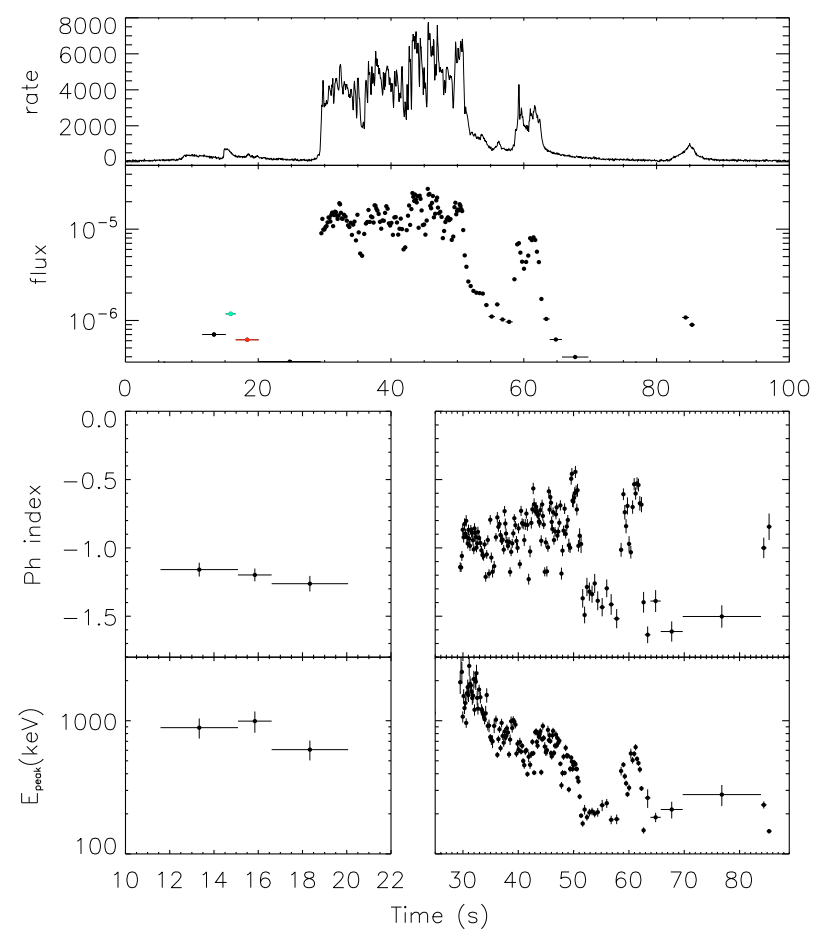

Fig. 26. Trigger \#3057. Color code and description as in Fig. 4a.

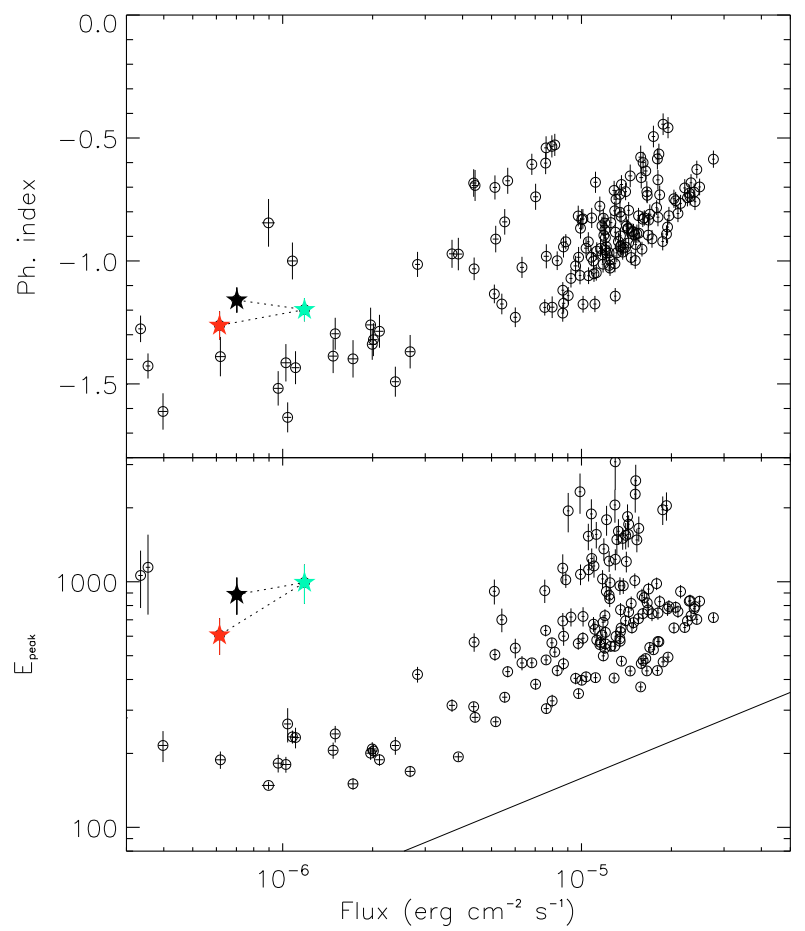

Fig. 27. Trigger \#3057. Color code and description as in Fig. 4b. 
D. Burlon et al.: Time resolved spectral behavior of bright BATSE precursors, Online Material p 6
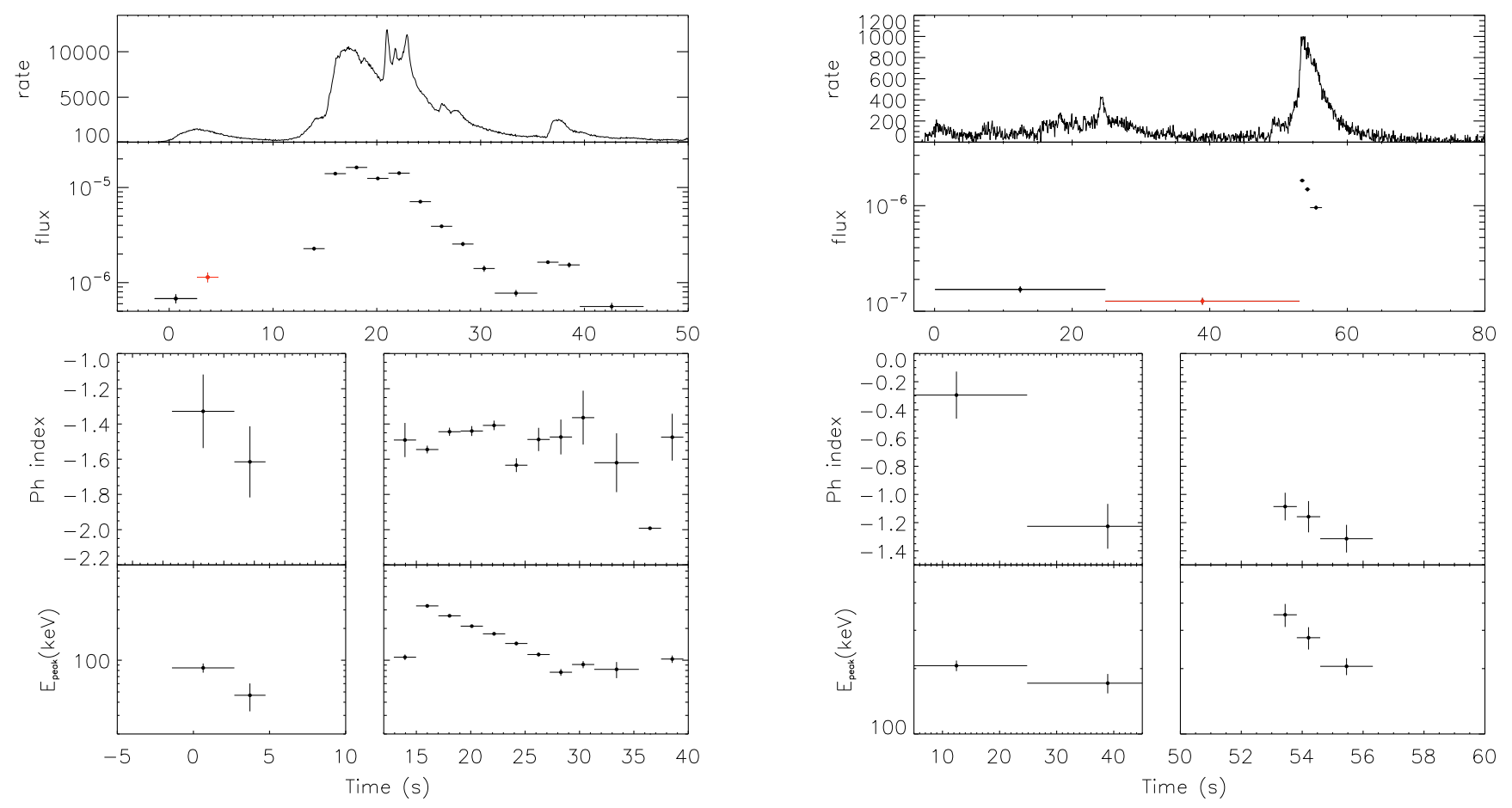

Fig. 28. Trigger \#4368. Color code and description as in Fig. 4a.
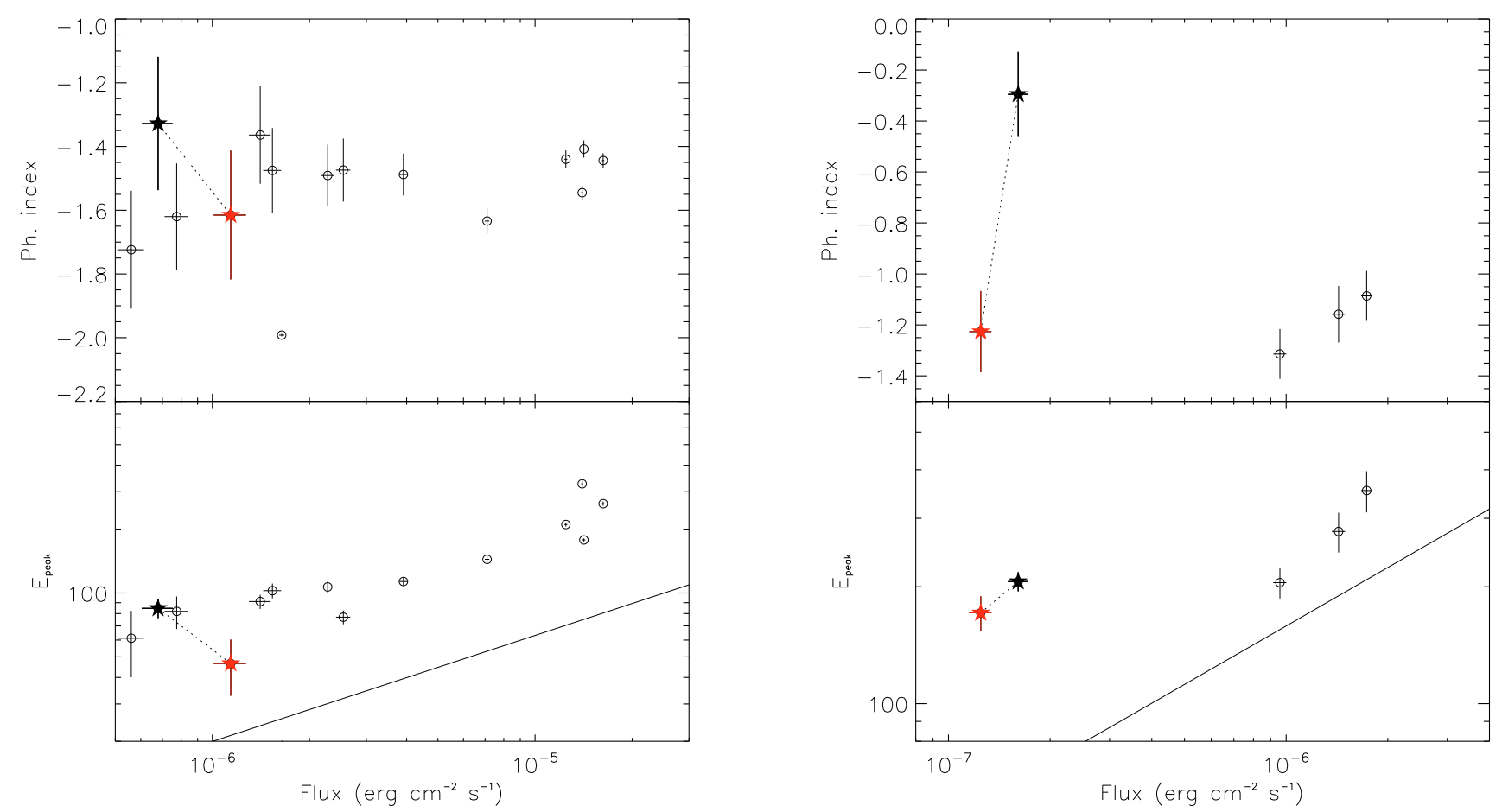

Fig. 29. Trigger \#4368. Color code and description as in Fig. 4b.

Fig. 31. Trigger \#2700. Color code and description as in Fig. 4b. 
D. Burlon et al.: Time resolved spectral behavior of bright BATSE precursors, Online Material p 7
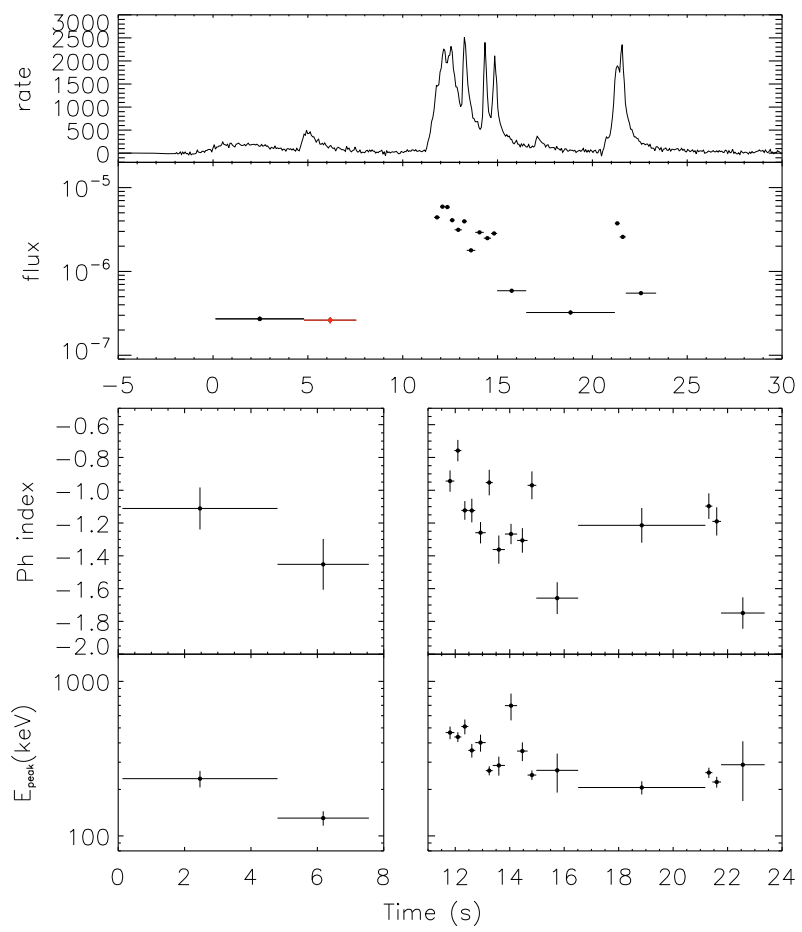

Fig. 32. Trigger \#1157. Color code and description as in Fig. 4a.

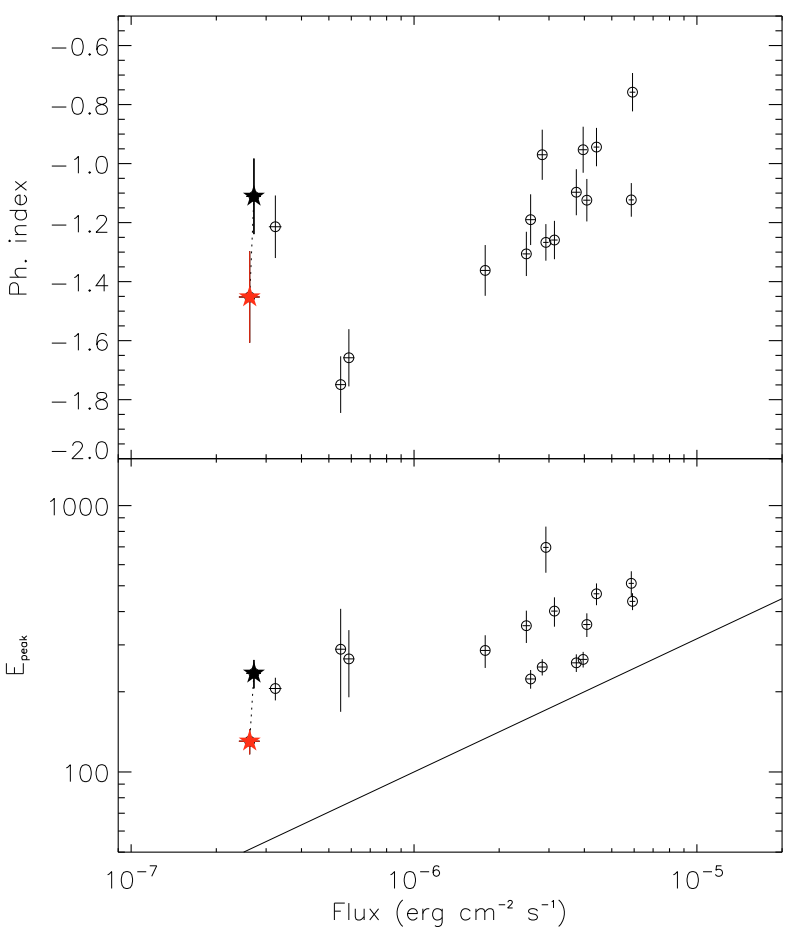

Fig. 33. Trigger \#1157. Color code and description as in Fig. 4b.

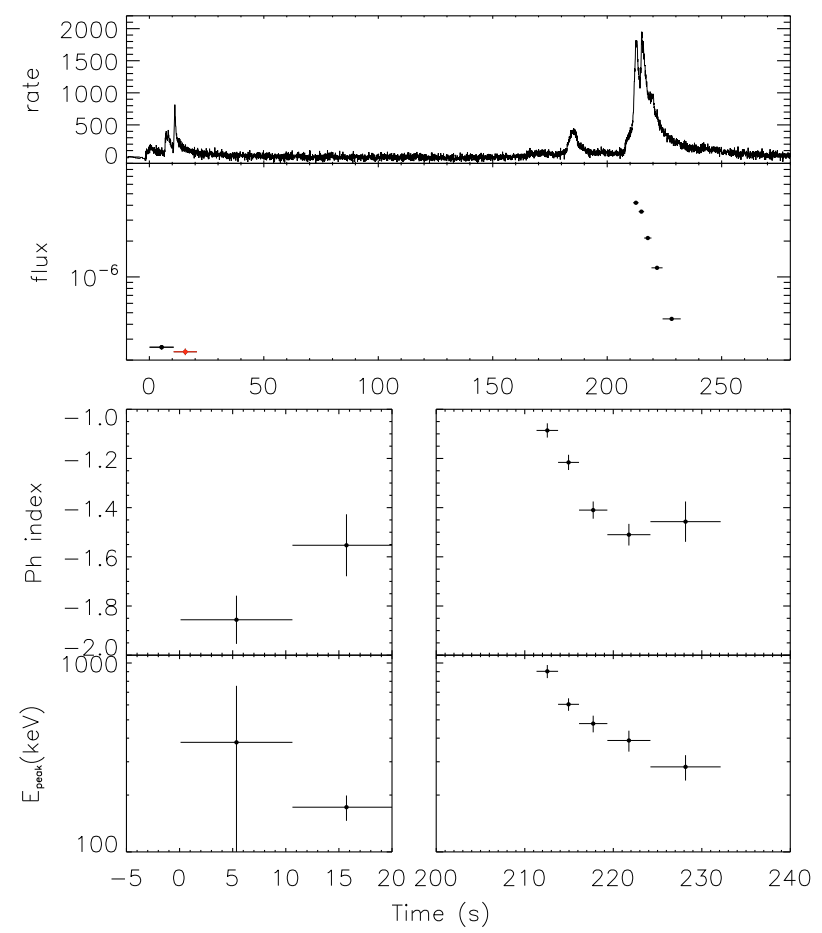

Fig. 34. Trigger \#6629. Color code and description as in Fig. 4a.

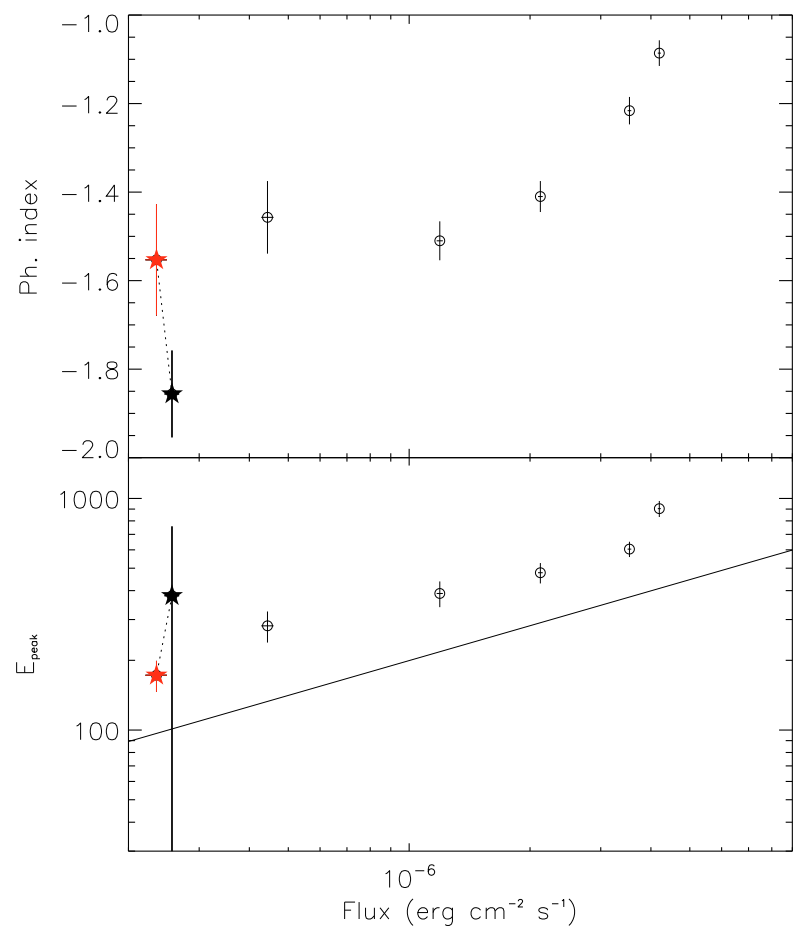

Fig. 35. Trigger \#6629. Color code and description as in Fig. 4b. 
D. Burlon et al.: Time resolved spectral behavior of bright BATSE precursors, Online Material $p 8$

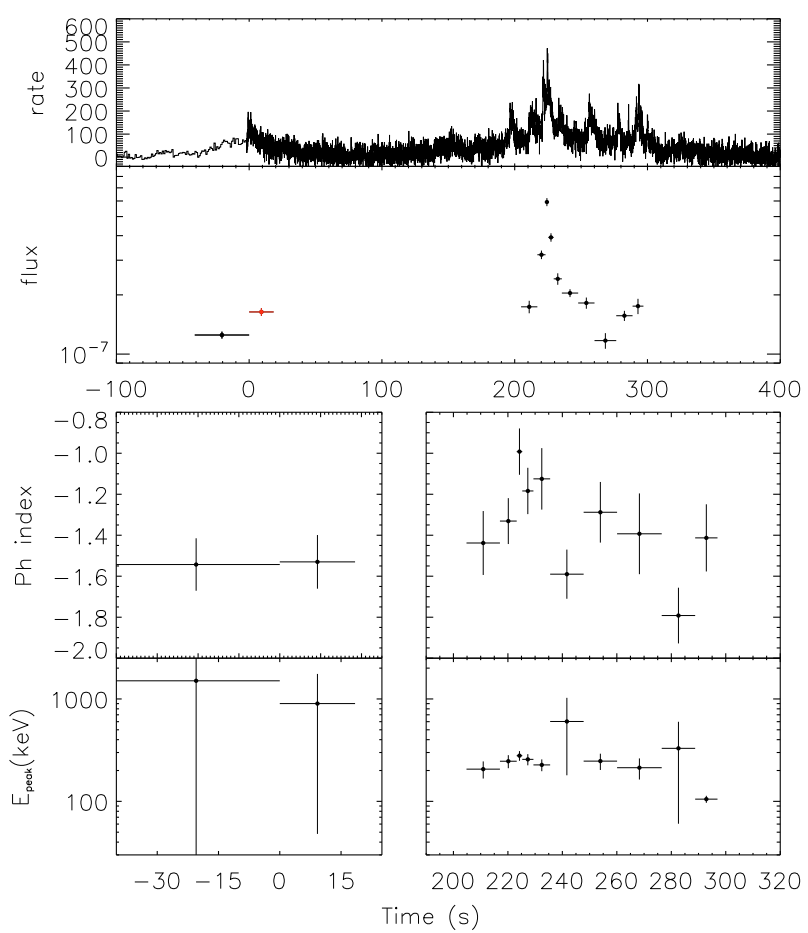

Fig. 36. Trigger \#3448. Color code and description as in Fig. 4a.

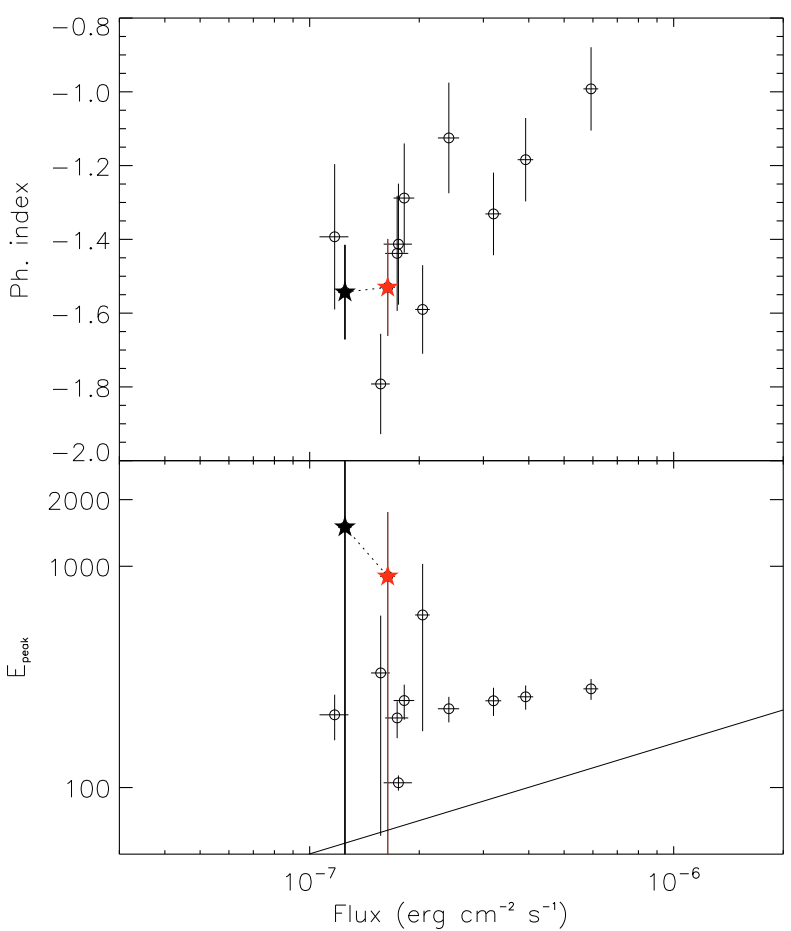

Fig. 37. Trigger \#3448. Color code and description as in Fig. 4b.

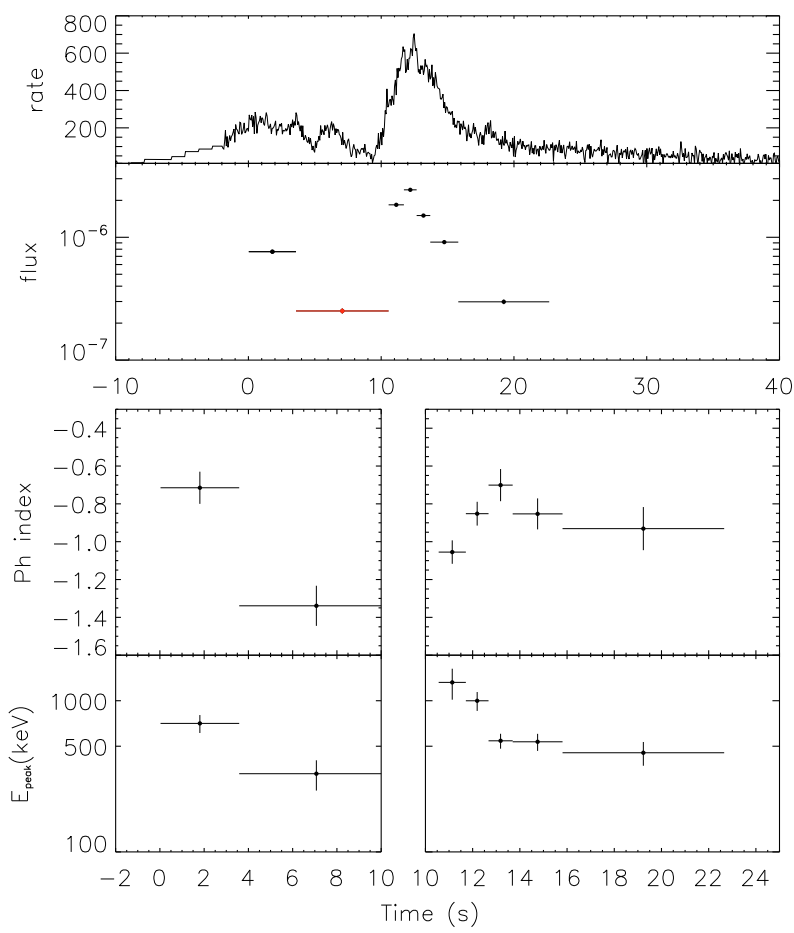

Fig. 38. Trigger \#3301. Color code and description as in Fig. 4a.

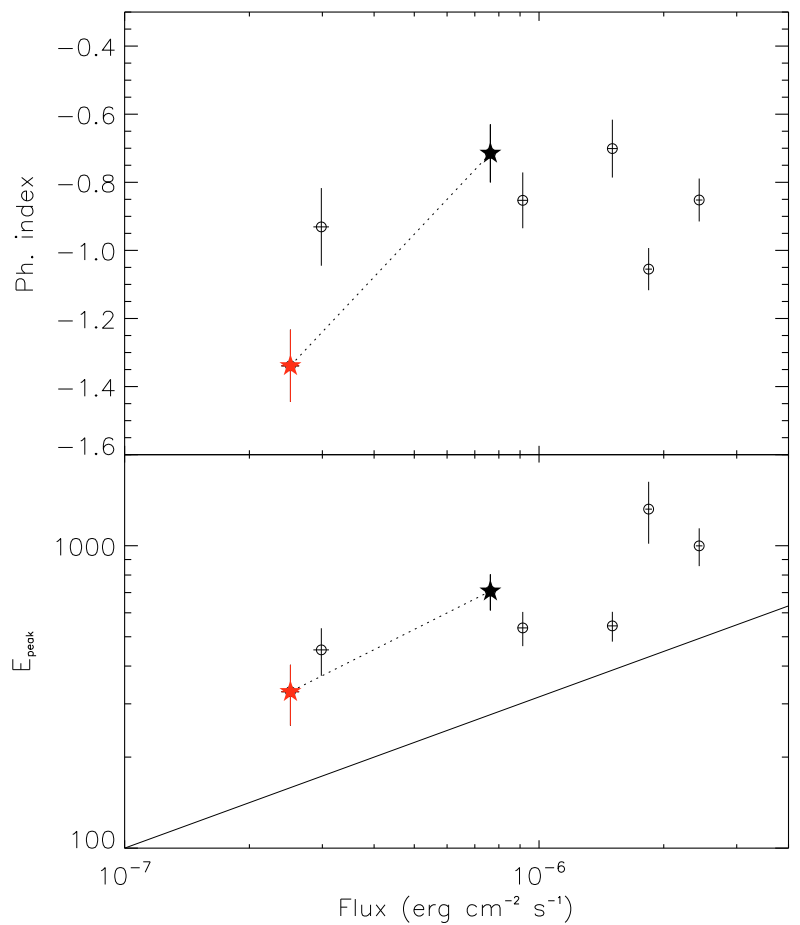

Fig. 39. Trigger \#3301. Color code and description as in Fig. 4b. 
D. Burlon et al.: Time resolved spectral behavior of bright BATSE precursors, Online Material $p 9$

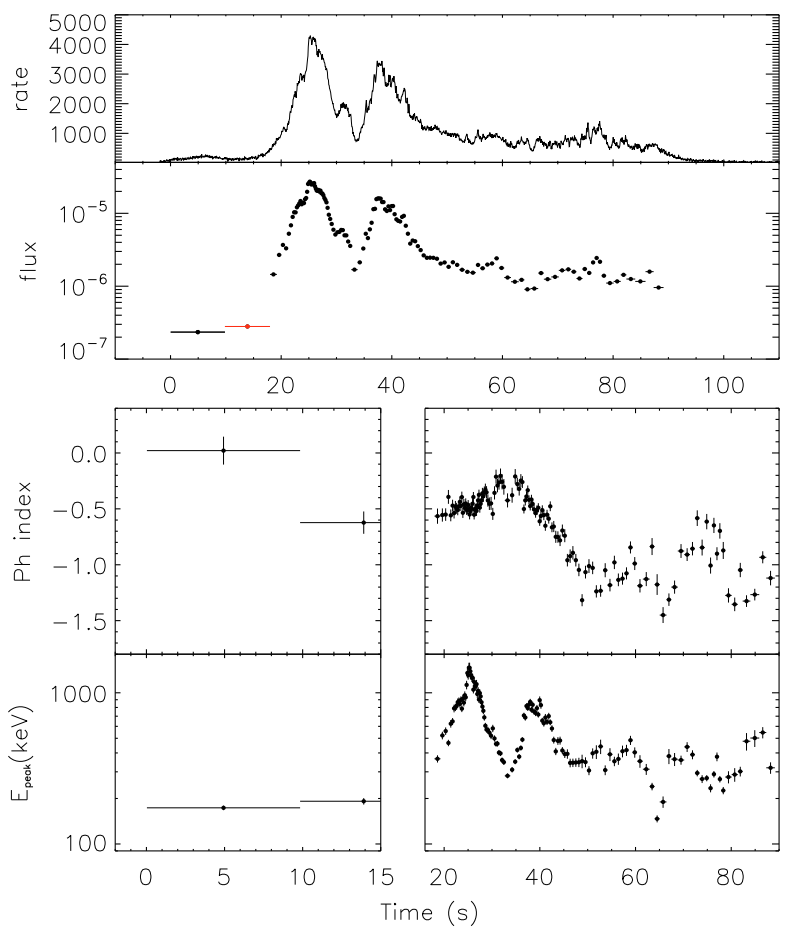

Fig. 40. Trigger \#7343. Color code and description as in Fig. 4a.

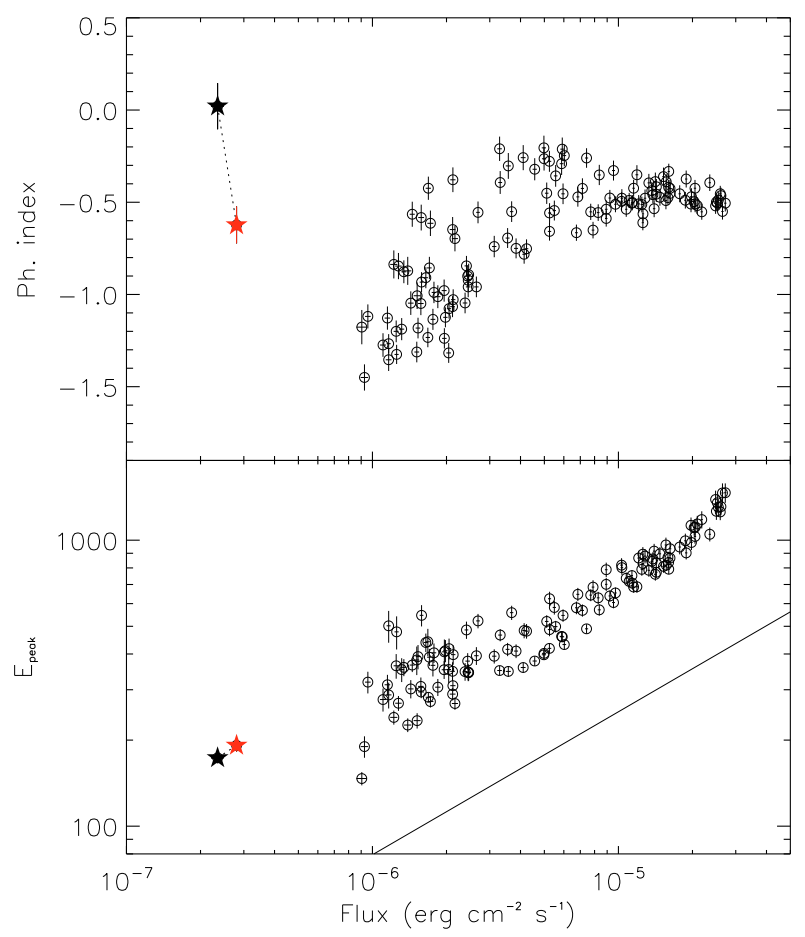

Fig. 41. Trigger \#7343. Color code and description as in Fig. 4b. 